ARTICLE

httpst//doi.org/10.1038/s414.67-020-15872-y

\title{
MALT-1 mediates IL-17 neural signaling to regulate $C$. elegans behavior, immunity and longevity
}

Sean M. Flynn (iD 1, Changchun Chen (iD 1,3, Murat Artan¹, Stephen Barratt' ${ }^{1}$, Alastair Crisp 1 , Geoffrey M. Nelson (1) 1,4, Sew-Yeu Peak-Chew², Farida Begum², Mark Skehel ${ }^{2} \&$ Mario de Bono (D) 1,5凶

Besides pro-inflammatory roles, the ancient cytokine interleukin-17 (IL-17) modulates neural circuit function. We investigate IL-17 signaling in neurons, and the extent it can alter organismal phenotypes. We combine immunoprecipitation and mass spectrometry to biochemically characterize endogenous signaling complexes that function downstream of IL-17 receptors in C. elegans neurons. We identify the paracaspase MALT-1 as a critical output of the pathway. MALT1 mediates signaling from many immune receptors in mammals, but was not previously implicated in IL-17 signaling or nervous system function. C. elegans MALT-1 forms a complex with homologs of Act1 and IRAK and appears to function both as a scaffold and a protease. MALT-1 is expressed broadly in the $C$. elegans nervous system, and neuronal IL-17-MALT-1 signaling regulates multiple phenotypes, including escape behavior, associative learning, immunity and longevity. Our data suggest MALT1 has an ancient role modulating neural circuit function downstream of IL-17 to remodel physiology and behavior.

\footnotetext{
${ }^{1}$ Cell Biology Division, Medical Research Council Laboratory of Molecular Biology, Cambridge CB2 OQH, United Kingdom. ${ }^{2}$ Biological Mass Spectrometry and Proteomics, Cell Biology Division, Medical Research Council Laboratory of Molecular Biology, Cambridge CB2 OQH, United Kingdom. ${ }^{3}$ Present address: Umeå Center for Molecular Medicine, Wallenberg Center for Molecular Medicine, Umeå University, SE-901 87 Umeå, Sweden. ${ }^{4}$ Present address: Department of Biomedical Informatics, Harvard Medical School, Boston, MA 02115, USA. ${ }^{5}$ Present address: Institute of Science and Technology Austria (IST

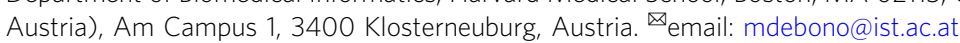


mmune signaling pathways can regulate the development and function of the nervous system in both health and disease ${ }^{1-3}$. Many of these effects are mediated by cytokines, small, secreted proteins that can participate in neuroimmune and interneuronal communication. For example, low levels of IL- $1 \beta$ and $\mathrm{TNF} \alpha$ regulate synaptic and homeostatic plasticity in healthy animals 4,5 ; pathological levels of proinflammatory cytokines during inflammation can disrupt fetal brain development, alter adult behavior ${ }^{6-9}$, and drive hyperalgesia and neuroinflammatory diseases ${ }^{10}$. Progression of neurodegenerative diseases, including Alzheimer's, Parkinson's and Amyotrophic lateral sclerosis (ALS), has also been associated with chronic inflammation ${ }^{11,12}$.

Recent work shows that the interleukin 17 (IL-17) proinflammatory cytokine can modify neural circuit activity. In a rodent model of infection during pregnancy, IL-17 secretion during maternal immune activation drives autism-related behaviors in the pups ${ }^{13}$. This phenotype is associated with hyperactivity of a specific cortical sub-region that expresses IL-17 receptors $(\mathrm{IL}-17 \mathrm{R})^{14}$. In mice, IL-17 can also lower the activation threshold of nociceptive neurons, and contributes to mechanical hyperalgesia ${ }^{15,16}$. In C. elegans IL-17Rs are expressed throughout the nervous system, and ILC-17.1 (interleukin cytokine 17 related 1), a homolog of mammalian IL-17s, has been shown to act on the RMG hub interneurons, increasing their response to presynaptic input from oxygen $\left(\mathrm{O}_{2}\right)$ sensors. The increased circuit gain conferred by ILC-17.1 enables $C$. elegans to persistently escapes $21 \% \mathrm{O}_{2}$, an aversive cue associated with surface exposure $^{17}$. Specific sensory responses and behaviors are thus modulated by IL-17 across distantly-related species, suggesting IL-17 has broad and conserved roles in regulating neuronal properties.

While IL-17's action on the nervous system is now established, its molecular effectors there are poorly understood. Moreover, the extent to which IL-17 signaling contributes to brain function and physiology is unclear, even in the well-defined C. elegans nervous system.

Here, we report that IL-17 signaling in the C. elegans nervous system is mediated by the paracaspase MALT-1. MALT1 is an ancient protein ${ }^{18}$ studied extensively, and almost exclusively, in the mammalian immune system. It is a key signaling molecule in innate and adaptive immunity, mediating signaling from ITAMcontaining (immunoreceptor tyrosine-based activation domain) receptors, including the $\mathrm{B}$-cell and T-cell receptors ${ }^{19-21}$. MALT1 has not been shown to mediate IL-17 signaling, but there has been speculation of such involvement. In situ hybridization suggests widespread MALT1 expression in mouse brain, (Allen Brain Atlas), but no physiological role in neurons has been reported. We find that C. elegans MALT-1 is expressed throughout the nervous system and forms an in vivo complex with IL-17 signaling components, namely the $C$. elegans homo-

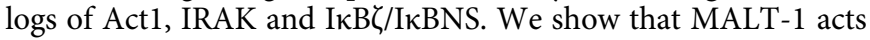
both as a protease and a scaffold to regulate neural function. Defects in IL-17/MALT-1 signaling lead to reconfigured gene expression, and changes in behavior and physiology, including altered immunity and extended lifespan.

\section{Results}

Proteomics identifies an ACTL-1-IRAK-MALT-1-NFKI-1 complex. C. elegans IL-17 signaling components appear to be expressed predominantly in the nervous system ${ }^{17}$. We epitope tagged all soluble IL-17 pathway components highlighted by genetics ${ }^{17}$, immunoprecipitated them from C. elegans extracts, and identified interacting proteins using mass spectrometry (MS, Fig. 1a).

ACTL-1 and PIK-1 are C. elegans orthologs of mammalian Act1 and IRAKs, respectively, and signal downstream of the
C. elegans IL-17 co-receptors ILCR-1 and ILCR-2 ${ }^{17}$. Genetic analysis suggests NFKI-1, a homolog of mammalian I $\mathrm{B} \zeta$ and I $\kappa$ BNS, acts downstream of ACTL-1, PIK-1, and ILCR-1/ILCR-2 co-receptors ${ }^{17}$.

We tagged endogenous ACTL-1 with a FLAG epitope, endogenous PIK-1 with a Myc epitope, and integrated an nfki$1:: g f p$ transgene. We showed the tagged proteins were functional (Supplementary Fig. 1), and immunoprecipitated them from C. elegans extracts. As controls, we immunoprecipitated proteins unrelated to IL-17 signaling tagged with the same epitopes. Using mass spectrometry (LC-MS/MS) we identified specific interactors for each signaling component (Fig. 1b-g and Supplementary Data $1 \mathrm{a}-\mathrm{c})$.

As expected from co-IP experiments using mammalian tissue culture cells $^{17}$, PIK-1 co-precipitated specifically with ACTL-1 (Fig. 1b), and reciprocally, ACTL-1 co-precipitated specifically with PIK-1 (Fig. 1c). IP of NFKI-1 also identified ACTL-1 and PIK-1/IRAK as specific interactors, suggesting these proteins form a complex in vivo (Fig. 1d). We identified other apparently specific interactors for each component. These are listed in Supplementary Data 1 as a resource.

The C. elegans ortholog of the paracaspase MALT1 consistently co-immunoprecipitated with each of ACTL-1, PIK-1 and NFKI-1 (Fig. 1b-g). MALT1 paracaspases are cysteine proteases with specificity for arginine residues ${ }^{23,24}$. Their caspase-like protease domain is highly conserved, as is their domain organization, which consists of an N-terminal death domain (DD) followed by 2-3 Ig (immunoglobulin)-like motifs that flank the paracaspase domain (Supplementary Fig. 2a) ${ }^{25}$. Mammalian MALT1 signals downstream of $\mathrm{B}$ cell, $\mathrm{T}$ cell, and other cell surface receptors containing an ITAM motif, and forms a filamentous complex called the CBM signalosome that contains a CARD domain protein, BCL10, and MALT1 ${ }^{19-21}$ (Supplementary Fig. 2b). The functions of MALT1 in the immune system are under intense scrutiny, but its roles elsewhere, and in invertebrates, have not been established.

To confirm the biochemical interactions of MALT-1 with C. elegans IL-17 signaling components, we expressed functional, GFP-tagged MALT-1 pan-neuronally, and identified interacting partners using IP/MS of extracts from the transgenic C. elegans strain. As a control, we performed IP/MS on extracts from strains expressing GFP-tagged neuronal proteins unrelated to IL-17 signaling. ACTL-1, PIK-1, and NFKI-1 each interacted specifically with MALT-1-GFP (Fig. 1h, i). We also identified other specific MALT-1 interactors (Supplementary Data 1d) including the C. elegans ortholog of mammalian SARM1, called TIR-1, which is implicated in the immune response $\mathrm{e}^{26,27}$, left/right asymmetry of an olfactory neuron ${ }^{28}$, and experience-dependent plasticity ${ }^{29}$. MALT-1 also interacted specifically with a large group of proteins implicated in RNA metabolism, including splicing factors and poly A binding proteins, suggesting it may localize to the nucleus or ribonucleoprotein particles (RNPs) (Supplementary Fig. 3).

MALT-1 promotes aggregation and escape from $21 \% \mathrm{O}_{2}$. MALT1 has not previously been implicated in IL-17 signaling or neural function. In C. elegans, ILC-17.1 signals through the ILCR1/ILCR-2 receptors on the RMG interneurons to increase RMG responsiveness to input from their pre-synaptic partner, the URX $\mathrm{O}_{2}$-sensing neurons (Fig. 1j). Increased RMG signaling enables C. elegans to strongly and persistently escape $21 \% \mathrm{O}_{2}$ and to aggregate $^{17,30,31}$. To probe the functional relevance of our proteomics data we sought malt-1 alleles in a collection of 583 strains isolated in a genetic screen for aggregation-defective mutants. This collection has been subjected to whole genome sequencing, and previously yielded IL-17 pathway mutants ${ }^{17}$. Four strains in 
a

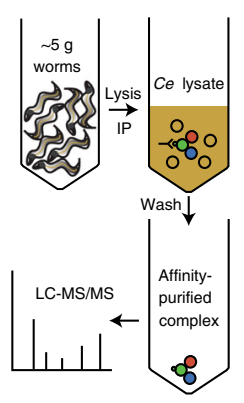

d

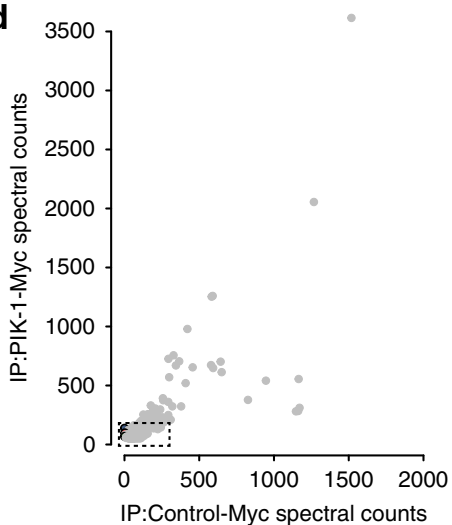

g

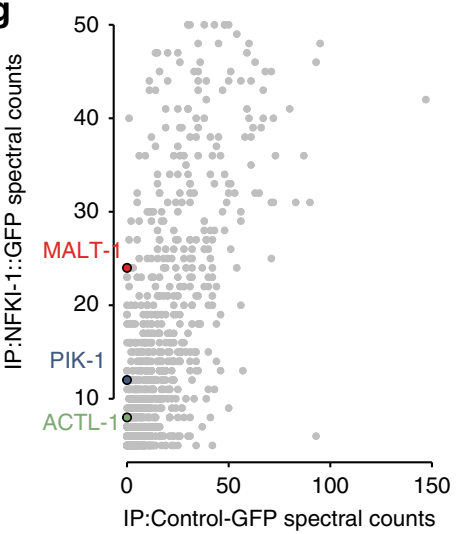

b

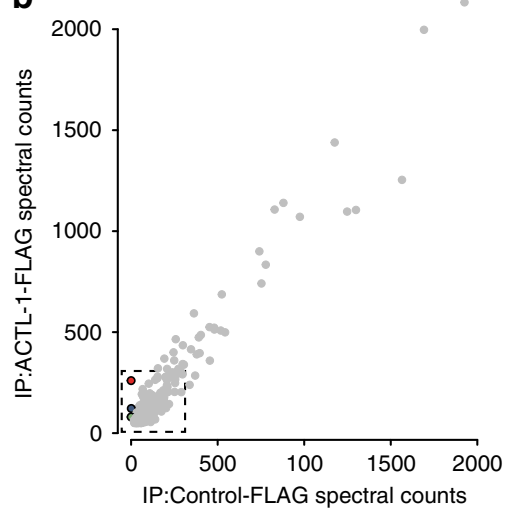

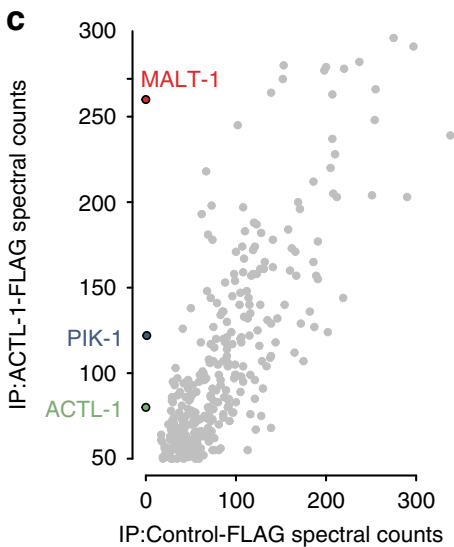

f

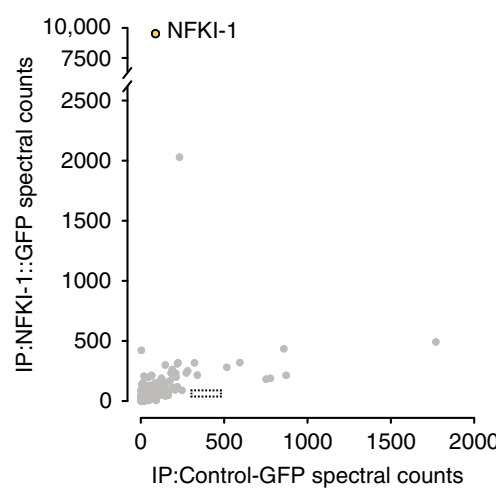

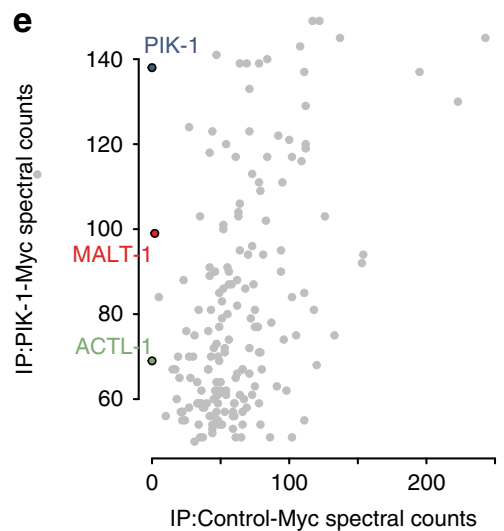
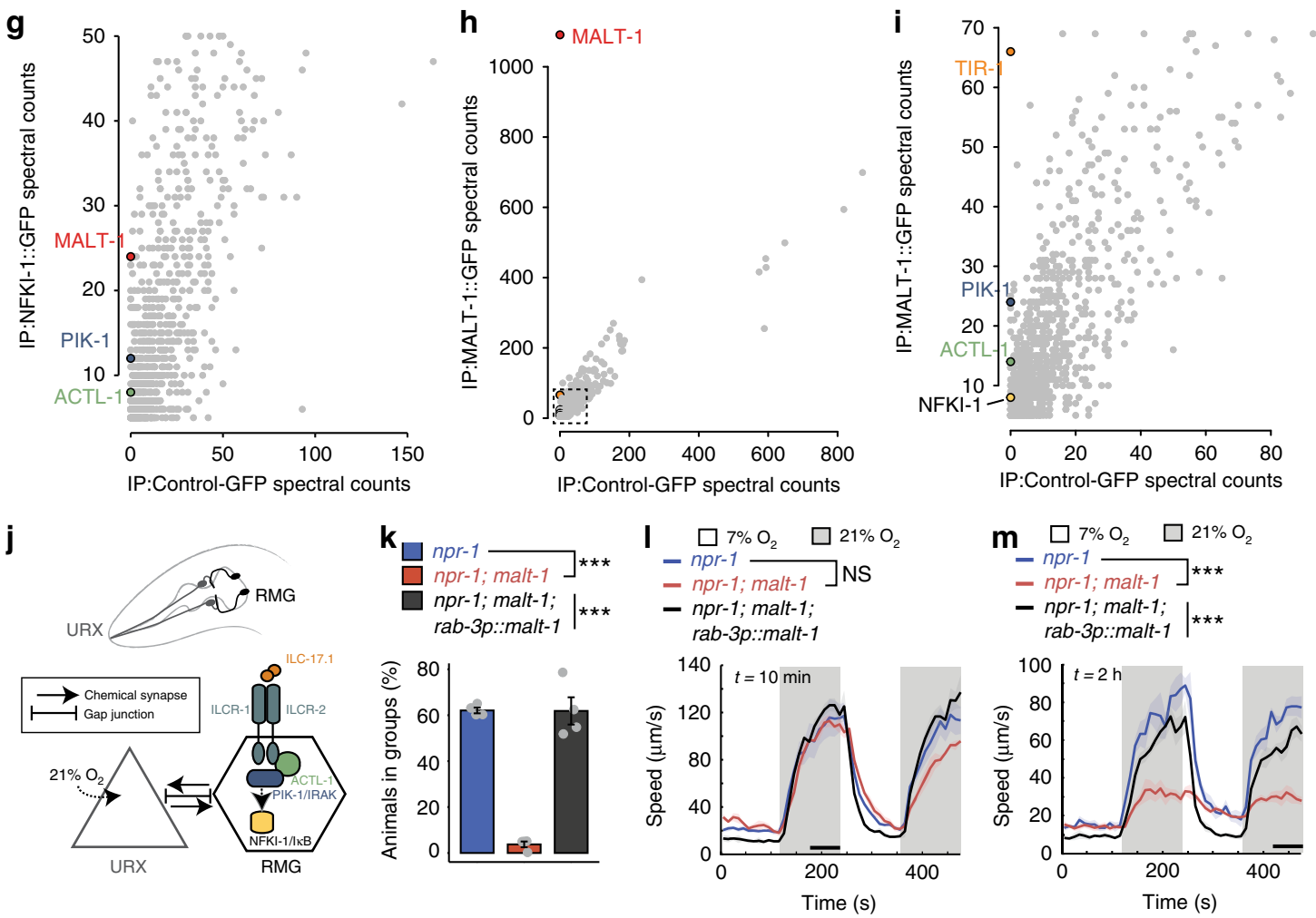

the collection harbored malt-1 alleles; one introduced a premature stop codon; another mutated the highly conserved E464 residue (Supplementary Fig. 4a and b), which is essential for catalytic activity in mammalian MALT1 ${ }^{32}$. We mapped the aggregation defect of this strain to an interval containing malt-1 (Supplementary Fig. 4c). Targeted disruption of malt-1 using
CRISPR/Cas9 resulted in an aggregation-defective strain whose phenotype could be rescued using a wild-type malt-1 transgene (Fig. 1k; and Supplementary Fig. 4f). These data confirm that MALT-1, like IL-17 signaling, promotes aggregation.

C. elegans aggregate to escape $21 \% \mathrm{O}_{2}$, a signal of surface exposure $^{33-35}$. In wild C. elegans isolates, $21 \% \quad \mathrm{O}_{2}$ evokes 
Fig. 1 MALT-1 forms a complex with ACTL-1, PIK-1/IRAK, and NFKI-1. a Schematic for affinity-purification and LC-MS/MS analysis of epitope-tagged IL17 signaling components from $C$. elegans extracts. $C e=C$. elegans. b-i Pull-down of ACTL-1-FLAG, PIK-1-Myc, or NFKI-1::GFP specifically co-IPs MALT-1 (b-g). Conversely, pull-down of MALT-1::GFP specifically co-IPS ACTL-1, PIK-1, and NFKI-1 (h and $\mathbf{i})$. Total spectral counts, a semi-quantitative readout of abundance ${ }^{22}$, are shown. $\mathbf{c}, \mathbf{e}, \mathbf{g}$, and $\mathbf{i}$ as in $\mathbf{b}, \mathbf{d}, \mathbf{f}$, and $\mathbf{h}$ except showing only the region marked by the black box in $\mathbf{b}, \mathbf{d}, \mathbf{f}$, and $\mathbf{h}$, respectively. $\mathbf{f}-\mathbf{i}$ Data is representative of two ( $\mathbf{f}$ and $\mathbf{g}$ ), or three (h and $\mathbf{i}$ ) biological replicates. $\mathbf{j}$ Schematic of IL-17 signaling in the $\mathrm{O}_{2}$-escape circuit. Increases in $\mathrm{O}_{2}$ levels are sensed by URX neurons, which tonically signal to RMG hub interneurons. IL-17 signaling increases the responsiveness of RMG neurons to promote escape from $21 \% \mathrm{O}_{2}$. $\mathbf{k}$ malt-1 promotes $\mathrm{C}$. elegans aggregation $\left(\mathrm{N}=4\right.$ assays). Data are presented as mean values $+/-\mathrm{SEM}$. ${ }^{\star \star \star} P<0.001$, one-way ANOVA with Tukey's post hoc HSD. I and $\mathbf{m}$ malt- 1 mutants are strongly aroused by $21 \% \mathrm{O}_{2}$ if stimulated immediately after transfer to the assay plate (I), but respond weakly to $21 \% \mathrm{O}_{2}$ if allowed to settle over a $2 \mathrm{~h}$ period $(\mathbf{m})$. I $n=86$ animals (npr-1), $n=46$ animals (npr-1; malt-1), $n=53$ animals ( $n$ pr-1; malt-1; rab-3p:: malt-1). $\mathbf{m} n=46$ animals (npr-1), $n=72$ animals (npr-1; malt-1), $n=46$ animals (npr-1; malt-1; rab-3p::malt-1). Plots show average speed (line) and SEM (shaded regions). Time of assay after transfer is shown at top left. NS, $P=0.8,{ }^{\star \star \star} P<0.001$, two-sided Mann-Whitney $U$ test. Here and in subsequent figures, black bars indicate time intervals used for statistical comparisons. See also Supplementary Figs. 1-4 and Supplementary Data 1.
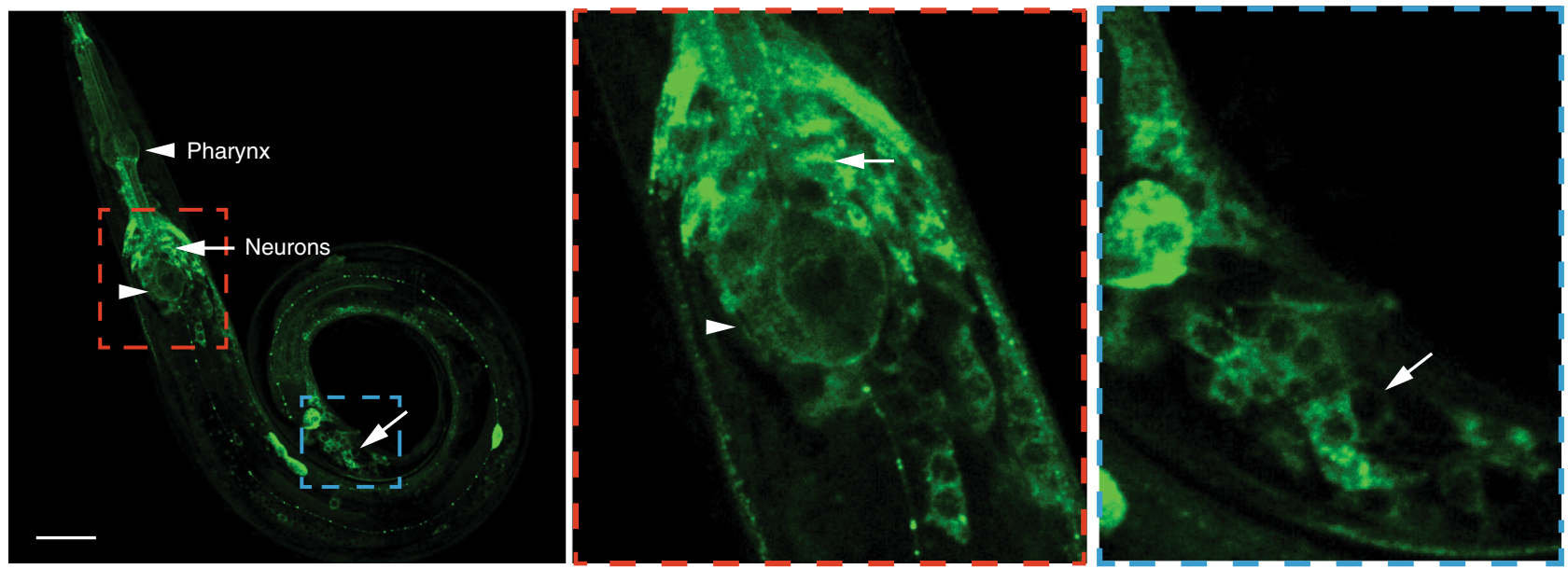

Fig. 2 MALT-1 is expressed widely in the nervous system. A transgene expressing C-terminally GFP-tagged MALT-1 from its endogenous promoter ( 4 kb of upstream DNA) is expressed broadly in the nervous system, including many neurons in the head (red box) and tail (blue box). MALT-1::GFP expression is also seen throughout the pharynx. Similar results were obtained in 3 experiments. White arrows point to neurons, arrowheads point to the pharyngeal bulbs. Scale bar: $20 \mu \mathrm{m}$. See also Supplementary Fig. 5.

sustained arousal ${ }^{36}$, a response also observed in $n p r-1$ (neuropeptide receptor 1) mutants of the domesticated N2 lab strain ${ }^{37}$. By contrast, npr-1 mutants defective in IL-17 signaling are not aroused by $21 \% \mathrm{O}_{2}$ in the absence of an additional arousal stimulus (e.g., being picked), and if this requirement is met, the arousal evoked by $21 \% \mathrm{O}_{2}$ is not sustained ${ }^{17}$. malt- 1 mutants showed these hallmark phenotypes (Fig. 1l, m), consistent with MALT-1 playing a role in C. elegans IL-17 signaling.

malt-1 mutants exhibited grossly normal growth rates, fertility, mating and feeding behaviors, and locomotion, although this was not quantitated. They exhibited a small but significant reduction in thrashing rate, suggesting a weak defect in locomotion (Supplementary Fig. 4e). Compared to their defects in escape from $21 \% \mathrm{O}_{2}$ however, this phenotype was relatively subtle.

MALT-1 modulates responsiveness of RMG interneurons to $\mathbf{O}_{2}$. malt-1::GFP and malt-1::RFP transgenes were expressed broadly in the nervous system (Fig. 2), including in the $\mathrm{O}_{2}$-sensing neurons AQR, PQR and URX (Supplementary Fig. 5) and their post-synaptic partner the RMG interneurons (Fig. 3a). malt1 phenotypes were rescued by expressing malt-1 cDNA panneuronally, confirming that MALT-1 has neuronal functions (Fig. 1k-m). Selectively expressing malt-1 cDNA in the RMG interneurons, or the $\mathrm{O}_{2}$-sensing neurons, restored aggregation behavior to malt-1 mutants (Fig. $3 \mathrm{~b}$ ), but only partially rescued the $\mathrm{O}_{2}$-response defects (Fig. $3 \mathrm{c}$ ). By contrast, we observed almost complete rescue of the $\mathrm{O}_{2}$ response phenotype when we expressed MALT-1 simultaneously in both sets of neurons (Fig. 3c;
Supplementary Fig. 4f and g). Thus, like ILCR-1 and ILCR-217, MALT-1 functions in RMG and AQR, PQR and URX to promote escape from $21 \% \mathrm{O}_{2}$.

$\mathrm{Ca}^{2+}$ imaging revealed that $\mathrm{O}_{2}$-evoked $\mathrm{Ca}^{2+}$ responses in RMG were significantly reduced in malt-1 mutants, both in immobilized (Fig. 4a) and freely moving (Supplementary Fig. 4h) animals. By contrast, $\mathrm{O}_{2}$-evoked $\mathrm{Ca}^{2+}$ responses in the URX sensory neurons appeared normal in malt-1 mutants (Fig. 4b). These phenotypes recapitulate those observed in IL-17 signaling mutants ${ }^{17}$. The $\mathrm{RMG} \mathrm{Ca}^{2+}$ response defect was rescued by expressing malt-1 cDNA from the $n p r-1$ promoter, which drives expression in RMG and the AQR, PQR and URX neurons (Fig. 4a). Together, these data indicate that, like ILCR-1 and ILCR-2, MALT-1 functions in both pre-synaptic and postsynaptic neurons in the $\mathrm{O}_{2}$-sensing circuit.

The malt-1 and ilc-17.1 mutant phenotypes were not additive. Both the $\mathrm{Ca}^{2+}$ signaling (Fig. 4c) and behavioral response (Fig. 4d) defects of malt-1; ilc-17.1 double mutants resembled those of single mutants, suggesting MALT-1 and ILC-17.1 function in the same pathway. Similarly, the RMG response defects of malt-1 mutants were not enhanced by defects in PIK-1/ IRAK (Supplementary Fig. 4h). Together, our biochemical, genetic, behavioral and physiological data suggest that the paracaspase MALT-1 mediates IL-17 signaling in neurons, most likely via a signaling complex made up of ACTL-1-IRAK/PIK1-MALT-1-NFKI-1.

To examine if malt- 1 is required developmentally, we expressed it selectively in adults using a heat-shock-inducible promoter. Without heat-shock, the phsp-16::malt-1 cDNA transgene did not 

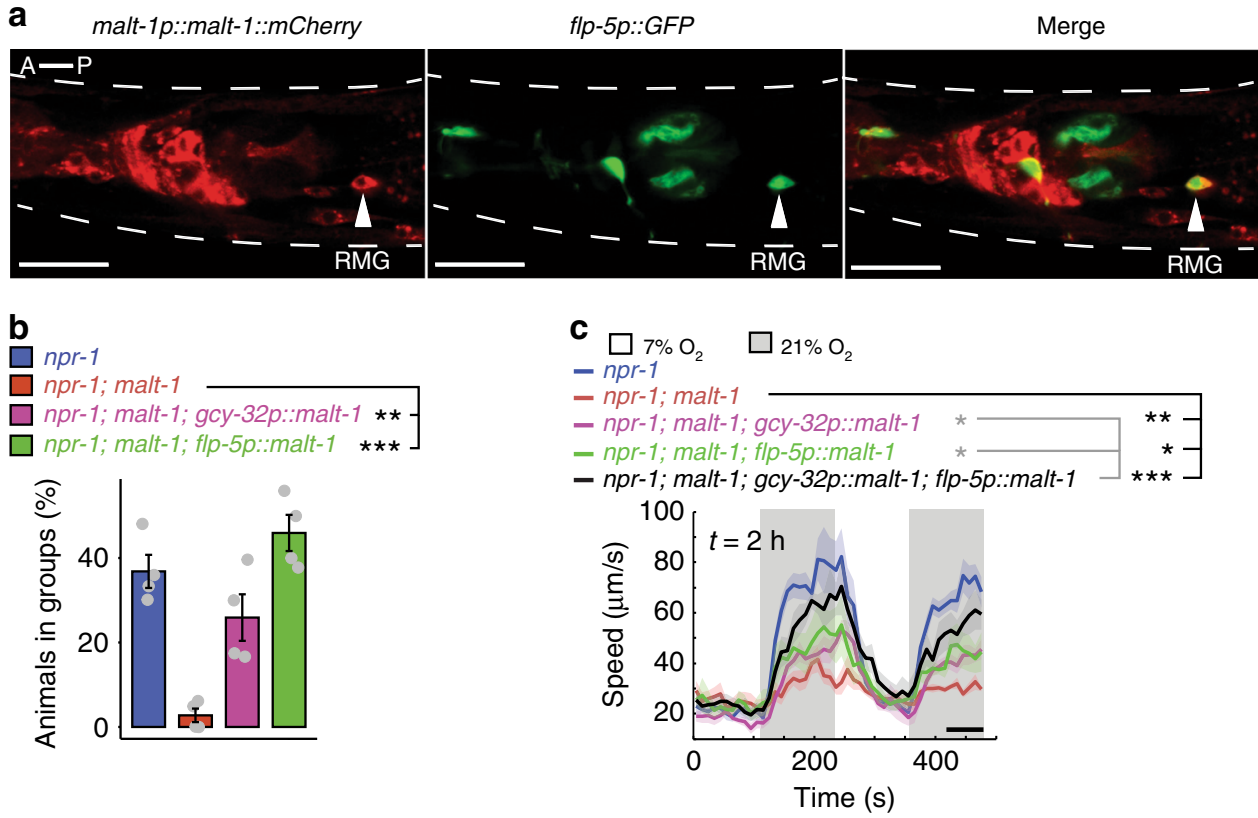

Fig. 3 MALT-1 functions in RMG interneurons. a A MALT-1::mCherry translational fusion, expressed from its endogenous promoter (4 kb), is expressed in RMG interneurons. RMG is recognized by its characteristic shape, location, and using a flp-5p::gfp reporter. Similar results were obtained in 3 experiments. Scale bars: $20 \mu \mathrm{m}$. b Expressing malt-1 cDNA from either the flp-5 promoter (RMG, ASG, PVT, 14, M4, and pharyngeal muscle), or the gcy-32 promoter (URX, AQR and PQR) rescues the aggregation defect of malt-1 mutants. $N=4$ assays. Data are presented as mean values $+/-\mathrm{SEM}$. ${ }^{\star \star} P<0.01,{ }^{\star \star \star} P<$ 0.001 , one-way ANOVA with Tukey's post hoc HSD. c The $\mathrm{O}_{2}$-response defect of malt-1 mutants is partially rescued by expressing malt-1 cDNA from the flp-5 promoter (RMG, ASG, PVT, 14, M4, and pharyngeal muscle), or the gcy-32 promoter (URX, AQR and PQR), and almost completely rescued when malt- 1 is expressed from both promoters simultaneously. Lines indicate average speed and shaded regions indicate SEM. $n=55$ animals $(n p r-1), n=85$ animals (npr-1; malt-1), $n=58$ animals (npr-1; malt-1; gcy-32p::malt-1), $n=66$ animals (npr-1; malt-1; flp-5p::malt-1), $n=46$ animals ( $n p r-1 ;$ malt-1; gcy-32p:: malt-1, flp-5p::malt-1). Plots show average speed (line) and SEM (shaded regions). ${ }^{\star} P<0.05$, ${ }^{\star \star} P<0.01,{ }^{\star \star \star} P<0.001$, two-sided Mann-Whitney $U$ test.

rescue the $\mathrm{O}_{2}$-response phenotype of malt-1 mutants (Fig. 4e). Heat-shock-induced expression during the $4^{\text {th }}$ larval stage was sufficient to restore behavioral responses (Fig. 4f), suggesting that MALT-1, like other IL-17 signaling components ${ }^{17}$, can alter circuit properties after the circuits have developed.

MALT-1 functions as a protease in the nervous system. In the mammalian immune system MALT1 functions both as a scaffold and as a protease. To examine if MALT-1 acts as a protease in neurons we edited the active site cysteine of the endogeneous malt-1 gene to alanine. The equivalent mutation is used in a paracaspase-dead model in mice ${ }^{38-40}$. malt-1 C374A animals resembled malt- 1 null mutants, and could be rescued by pan-neuronal expression of malt-1 cDNA (Fig. 5a; Supplementary Fig. 6a). By contrast, a malt-1 C374A transgene was unable to rescue the phenotype of malt-1(db1194) mutants (Fig. 5b). Unexpectedly, overexpressing malt-1 C374A in a WT background conferred a malt-1(null) phenotype (Fig. 5c), suggesting that catalytically dead MALT-1 can act as a dominant negative. Together these data suggest that MALT-1 protease activity is important for its function in the C. elegans nervous system.

We also asked if IL-17 signaling requires PIK-1/IRAK kinase activity. We created a single copy transgene in which the ATPbinding pocket lysine residue (K217) of PIK-1 was mutated to alanine. The K217A transgene rescued pik-1(null) phenotypes (Supplementary Fig. 6b), suggesting that kinase activity is not essential for PIK-1 to regulate behavior.

MALT-1 promotes assembly of IL-17 signaling complexes. To extend our in vivo proteomic analyses we made a strain in which endogenous ACTL-1, PIK-1, MALT-1, and NFKI-1 were each tagged with different epitopes. To corroborate our LC-MS/MS data we first showed that ACTL-1, PIK-1, and NFKI-1 specifically co-immunoprecipitated with MALT-1 in a multiple knock-in strain (Fig. 6a).

To analyze the signaling complex further we carried out IPs from strains overexpressing NFKI-1-GFP. When we quantitatively compared NFKI-1 complexes from WT, malt- 1 and pik-1 mutants, using IP/MS, we found that the amount of PIK-1/IRAK co-precipitating with NFKI-1 was reduced when MALT-1 was absent (Fig. 6b). By contrast, in pik-1 mutants the interaction between MALT-1 and NFKI-1 was not significantly reduced (Fig. 6c). These data suggest that NFKI-1 recruitment to the signaling complex requires MALT-1.

To ask if MALT-1 and NFKI-1 interact directly, we expressed epitope-tagged versions of the proteins in E. coli, and performed pairwise tests for co-immunoprecipitation. MALT-1-HA immunoprecipitated NFKI-1-V5, and conversely NFKI-1-V5 immunoprecipitated MALT-1, supporting a direct physical interaction (Fig. 6d). MALT-1 also interacted directly with ACTL-1 (Fig. 6e).

Sub-domains of NFKI-1 and MALT-1 did not express well in E. coli. We therefore used the yeast two-hybrid assay to map domains mediating the interaction between MALT-1 and NFKI1. We found that the DD of MALT-1 could interact with the Nterminal half of NFKI-1 (Fig. 6f), suggesting that MALT-1's DD contributes to NFKI-1 binding.

Sub-cellular localization of IL-17 signaling components. In the mammalian immune system IRAKs and MALT1 are core components of the Myddosome and CBM signalosome, respectively. These complexes are structurally related filamentous oligomers that assemble in the cytosol ${ }^{41,42}$. I $\kappa$ B family proteins can perform 


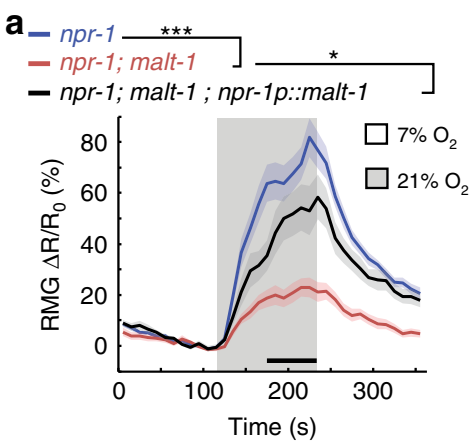

d

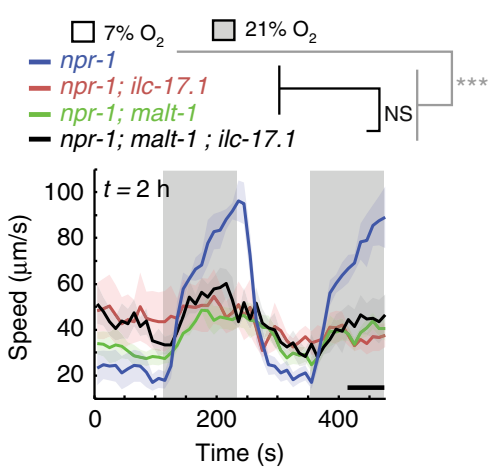

b
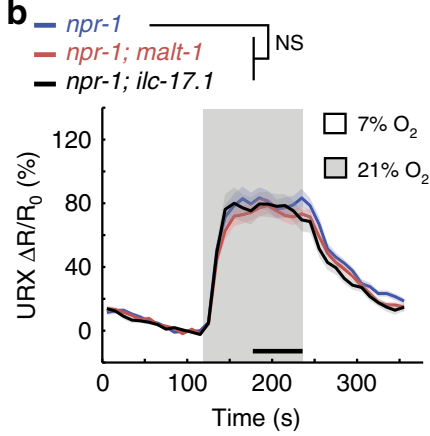

e
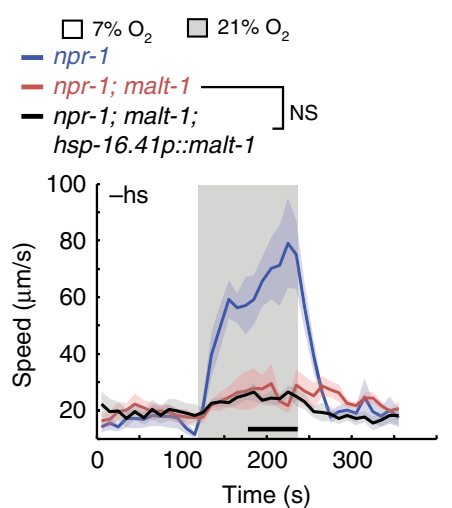

c

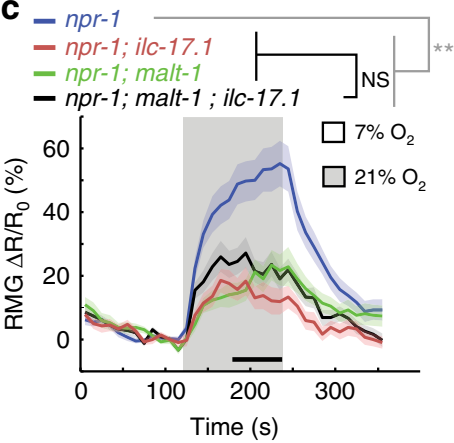

f $\left.\begin{array}{rl} & \square 7 \% \mathrm{O}_{2} \quad \square 21 \% \mathrm{O}_{2} \\ - & n p r-1 \\ - & n p r-1 ; \text { malt-1 } \\ - & \text { npr-1; malt-1; } \\ & \text { hsp-16.41p::malt-1 }\end{array}\right]_{\star \star *}$

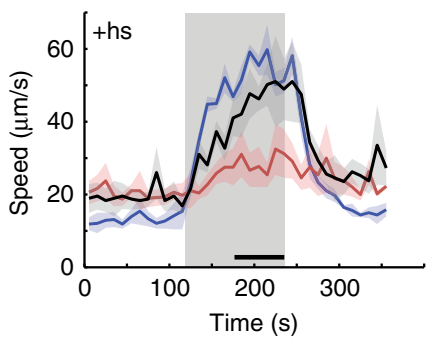

Fig. 4 MALT-1 mediates IL-17 signaling. a and b Disrupting malt-1 attenuates $\mathrm{Ca}^{2+}$ responses evoked by $21 \% \mathrm{O}_{2}$ in $\mathrm{RMG}$ (a) but not URX (b) neurons. The RMG defect can be rescued by expressing malt-1 cDNA in both RMG and URX, using the npr-1 promoter (a). a $n=25$ animals ( $n p r-1), n=35$ animals ( $n p r-1$; malt-1), $n=24$ animals (npr-1; malt-1; $n p r-1 p:: m a l t-1) . \mathrm{b} \mathrm{n}=20$ animals ( $n p r-1), \mathrm{n}=21$ animals (npr-1; malt-1), $\mathrm{n}=13$ animals (npr-1; ilc-17.1). Ca ${ }^{2+}$ responses are reported by YC2.60 cameleon. Lines indicate average speed and shaded regions indicate SEM. $\mathrm{n}=20$ animals $(n p r-1), n=21$ animals (npr-1; malt-1), $n=13$ animals (npr-1; ilc-17.1). ${ }^{\star} P=0.03,{ }^{\star \star \star} P=0.0003$, two-sided Mann-Whitney $U$ test. $c$ and d Null mutations in malt-1 and ilc-17.1 do not show additive phenotypes when either RMG Ca${ }^{2+}$ transients (c) or speed responses evoked by $21 \% \mathrm{O}_{2}$ are measured (d). Lines indicate average speed and shaded regions indicate SEM. $\mathbf{c} n=29$ animals ( $n$ pr-1), $n=25$ animals ( $n$ pr-1; malt-1), $n=28$ animals (npr-1; ilc-17.1), $n=31$ animals ( $n p r-1 ;$ malt-1; ilc-17.1). d $n=50$ animals (npr-1), $n=61$ animals ( $n p r-1 ;$ malt-1), $n=67$ animals (npr-1; ilc-17.1), $n=52$ animals (npr-1; malt-1; ilc-17.1). ${ }^{\star \star} p<0.01$, ${ }^{\star \star \star} p<0.001$, two-sided Mann-Whitney $U$ test. e A transgene expressing malt-1 cDNA from the $h s p-16.41$ promoter does not rescue malt-1 phenotypes in the absence of heat-shock. Plots show average speed (line) and SEM (shaded regions). $n=35$ animals (npr-1), $n=59$ animals ( $n p r-1 ;$ malt-1), $n=47$ animals (npr-1; malt-1; hsp-16.41p:: malt-1). $P=0.14$, two-sided Mann-Whitney $U$ test. $\mathbf{f}$ Heat-shock-induced cDNA expression in adults restores $\mathrm{O}_{2}$-evoked responses to malt-1 mutants. Plots show average speed (line) and SEM (shaded regions); $n=36$ animals ( $n p r-1), n=48$ animals (npr-1; malt-1), $n=33$ animals (npr-1; malt-1; hsp-16.41p::malt1). Plots show average speed (line) and SEM (shaded regions). ${ }^{\star \star \star} P=8.77 \mathrm{e}-06$, two-sided Mann-Whitney $U$ test.

both cytoplasmic and nuclear functions downstream of signalosome assembly ${ }^{43}$. Fractionation of a C. elegans lysate by gel filtration revealed that ACTL-1 and PIK-1 exist mostly as highmolecular weight species; they eluted in the heaviest fractions, including the void, of a gel filtration column (Fig. 6g and Supplementary Fig. 7). MALT-1 and NFKI-1 ran mostly as smaller species $(\sim 50-200 \mathrm{kDa})$, but they were also detectable in the heavier ACTL-1-containing and PIK-1-containing fractions. The high-molecular weight species we observed may be an artifact of unsolubilized membrane or protein aggregation, or may represent interactions with additional proteins. Alternatively, they may report oligomeric complexes of ACTL-1/PIK-1/MALT-1 related to the Myddosome and the CBM signalosome ${ }^{41,42}$, although this hypothesis requires further testing.

To determine the sub-cellular localization of IL-17 signaling components, we separated the nuclear and cytosolic fractions of our lysate. ACTL-1-FLAG and MALT-1-HA were consistently detected in both cytoplasmic and nuclear fractions (Fig. 7a). NFKI-1-V5 was predominantly in nuclear fractions (Fig. 7a; five replicates), although as NFKI-1-V5 immunoreactivity in the fractions was weak we cannot rule out the possibility that NFKI-1 was also present in the cytoplasmic fractions at levels below our detection threshold. It is notable that NFKI-1 specifically coimmunoprecipitated with transcription factors and chromatin state modifiers, including CREB binding protein (CBP), a histone acetyltransferase ${ }^{44}$, suggesting that NFKI-1 regulates transcription (Supplementary Data 1c).

MALT-1 and NFKI-1 provide partially parallel IL-17 outputs. Overexpressing NFKI-1 suppresses ilcr-1, actl-1 and pik-1 null phenotypes, suggesting NFKI-1 functions downstream of those signaling components ${ }^{17}$. Overexpressing MALT-1 also rescued the $\mathrm{O}_{2}$ arousal defects of ilc-17.1, ilcr-1 actl-1, and pik-1 mutants (Fig. 7b and c). To test whether MALT-1 functions upstream or downstream of NFKI-1, we asked whether overexpressing either component rescued a null mutant of the other. Overexpressing NFKI-1 in malt-1(null) mutants, or MALT-1 in nfki-1(null) animals, fully rescued the aggregation defect but either did not restore, or only partly restored, the arousal response to $21 \% \mathrm{O}_{2}$ (Fig. $7 \mathrm{~d}-\mathrm{g}$ ). These data suggest MALT-1 and NFKI-1 provide partially parallel outputs for IL-17 signaling.

Disrupting IL-17 signaling reprograms gene expression. In mammalian tissues IL-17 acts globally to drive pro-inflammatory gene expression ${ }^{45}$. We defined a transcriptional fingerprint of $C$. elegans IL-17 signaling by comparing the whole-animal RNA-seq profiles of ilc-17.1, malt-1, and nfki-1 mutants to that of controls 

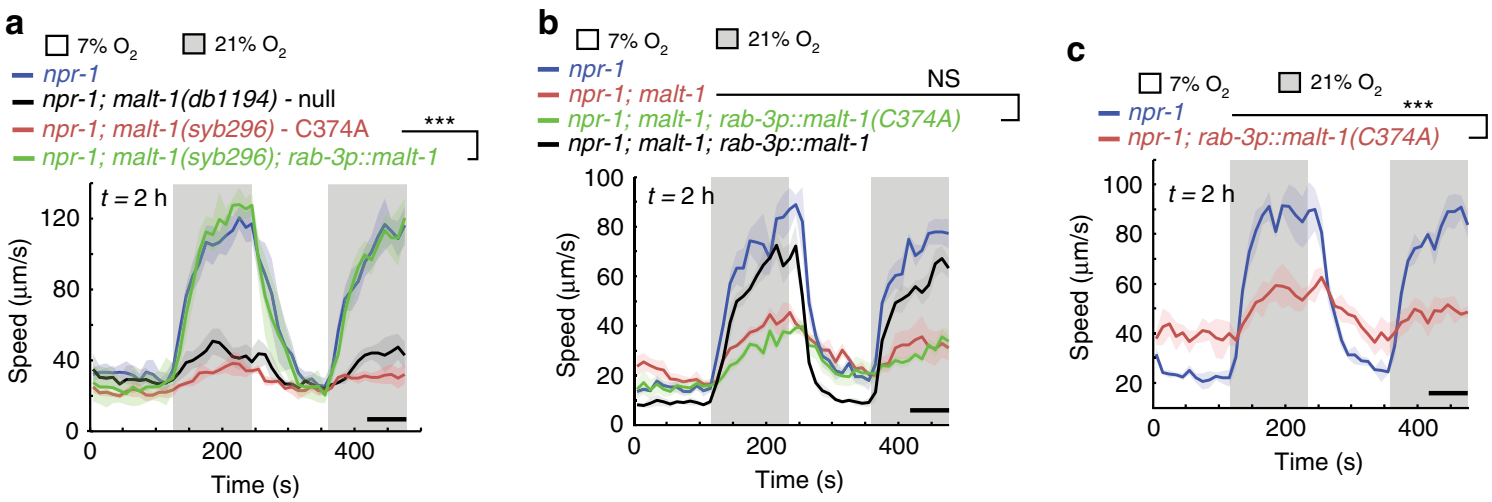

Fig. 5 MALT-1 has enzymatic roles in IL-17 signaling. a-c MALT-1's function in the nervous system requires its protease active site. a malt-1(syb296) mutants that express a catalytically inactive MALT-1 (C374A) show $\mathrm{O}_{2}$ response defects comparable to those of malt-1(null) animals. Pan-neuronal expression of malt-1 cDNA rescues this phenotype. $n=53$ animals ( $n p r-1), n=55$ animals ( $n$ pr-1; malt-1(db1194)), $n=50$ animals ( $n p r-1$; malt-1(syb296)), $n=29$ animals ( $n p r-1 ;$ malt-1(syb296); rab-3p::malt-1). Plots show average speed (line) and SEM (shaded regions). ${ }^{\star \star \star} P=2.95 \mathrm{e}-09$, two-sided MannWhitney $U$ test. b cDNA encoding a MALT-1 C374A catalytically inactive protein, expressed from the rab-3 promoter, does not rescue the $\mathrm{O}_{2}$ response defects of malt-1 mutants. Data corresponding to npr-1 and npr-1; malt-1; rab-3p::malt-1 in $\mathbf{b}$ are the same as those shown in Fig. $1 \mathrm{~m}$, and were obtained in parallel to the genotypes shown. $n=46$ animals ( $n p r-1), n=74$ animals ( $n p r-1 ;$ malt-1), $n=71$ animals ( $n$ pr-1; malt-1; rab-3p::malt-1(C374A)), $n=46$ animals (npr-1; malt-1; rab-3p::malt-1). Plots show average speed (line) and SEM (shaded regions). NS, $P=0.693918$, two-sided Mann-Whitney $U$ test. c

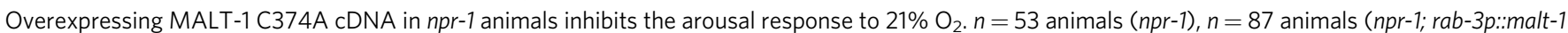
(C374A). Plots show average speed (line) and SEM (shaded regions). ${ }^{\star \star} P=1.09 e-12$, two-sided Mann-Whitney $U$ test. See also Supplementary Fig. 6.

(Supplementary Data 3). Data analysis suggested that pathways implicated in neuropeptide signaling, metabolism, ageing, and immunity were significantly altered by IL-17 signaling (Fig. 8a and $b$, and Supplementary Data 3).

To extend our analysis, we compared our dataset to a previous study that identified genes differentially expressed in animals acclimated to $21 \%$ and $7 \% \mathrm{O}_{2}{ }^{31}$. Most of the neuropeptides regulated by $\mathrm{IL}-17$ were not regulated by $\mathrm{O}_{2}$ experience ${ }^{31}$ (Supplementary Data 4), suggesting IL-17 elicits transcriptional changes not explained by altered activity of the $\mathrm{O}_{2}$-sensing circuit. These data suggest that IL-17 signaling may directly or indirectly alter many features of C. elegans behavior and global physiology.

MALT-1 and IL-17 signaling regulate multiple behaviors. The widespread expression of MALT-1 and other IL-17 signaling components in the nervous system, together with our RNA Seq data, suggested that IL-17 signaling forms an important neuromodulatory axis in C. elegans. To begin probing this hypothesis we tested mutants in an associative learning paradigm. In this assay animals associate an environment high in $\mathrm{NaCl}$ with food withdrawal, which leads them to suppress salt attraction when subsequently tested in a chemotaxis assay ${ }^{46}$. Mutants in ilc-17.1, pik-1, and $n f k i-1$ exhibit normal naive responses to salt ${ }^{17}$. By contrast, all IL-17 signaling mutants we tested retained stronger attraction to salt than controls after conditioning (Fig. 8c). IL-17 and MALT-1 therefore regulate associative learning, as well as escape from $21 \%$ $\mathrm{O}_{2}$. We could rescue the ilcr-1 learning phenotype by selectively expressing cDNA encoding the ILCR-1 receptor in the ASE saltsensing neurons $(f l p-6 p)$, but not in the RMG interneurons $(f l p-5 p)$ or the $\mathrm{O}_{2}$ sensors $(g c y$-32p) (Fig. $8 \mathrm{~d})$, indicating that IL-17 signaling in the nervous system is not restricted to the $\mathrm{O}_{2}$-sensing circuit.

Neural IL-17-MALT-1 signaling alters immunity and lifespan. We next assessed the impact of MALT-1 signaling on physiological phenotypes known to be regulated by the nervous system. To explore immune functions, we measured survival on Pseudomonas aeruginosa, a bacterial pathogen that colonizes the intestine of C. elegans $^{47}$. We carried out these experiments in animals having the $\mathrm{N} 2$ version of the $n p r-1$ neuropeptide receptor, $n p r-1215 \mathrm{~V}$, which inhibits aggregation behavior and escape from $21 \% \mathrm{O}_{2}{ }^{34,48}$; this ensures differences in hyperoxia avoidance do not contribute to altered pathogen resistance. To further exclude behavioral effects, we tested survival on PA14 using both small lawn assays, in which animals are able to avoid the pathogen, and big lawn assays, in which they are not ${ }^{49}$.

Animals lacking malt-1, or harboring the malt-1 protease-dead allele malt-1(C374A), were resistant to PA14 infection compared to N2 controls in both small lawn (Fig. 6e and f) and big lawn assays (Supplementary Fig. 8a and b). PA14 resistance in malt-1 (null) mutants was rescued by pan-neuronal expression of malt-1 cDNA (Fig. 8e and Supplementary Fig. 8a), suggesting that MALT-1 acts in the nervous system to regulate the immune response. Like malt-1 mutants, ilcr- 1 and $n f k i-1$ mutants survived significantly longer on PA14 than controls (Fig. 8g, h and Supplementary Fig. 8c, d).

Increased pathogen resistance is often associated with increased lifespan. To examine if disrupting IL-17 signaling alters lifespan we measured survival on the standard laboratory food source of C. elegans, E. coli OP50. ilc-17.1 and malt-1 mutants lived significantly longer than N2 controls (Fig. 8j, k). Expression of ilc-17.1 cDNA from its endogenous promoter not only rescued the phenotype of the null mutant, but significantly reduced lifespan compared to non-transgenic N2 controls (Fig. 8k). We could rescue the extended lifespan of malt-1 mutants by pan-neuronal expression of malt-1 (Fig. 8j), suggesting that IL-17 signaling acts in the nervous system to regulate longevity. The lifespan phenotypes of malt-1 and ilc-17.1 mutants were not additive (Fig. 81). Furthermore, the ability of ILC-17.1 overexpression to reduce lifespan was dependent on malt-1 (Fig. $8 \mathrm{~m}$ ). Together, these two observations suggest that MALT-1 acts downstream of ILC-17.1 to negatively regulate longevity.

MALT-1 strongly and specifically co-immunoprecipitated with factors known to regulate longevity or immunity, including NHR$49^{50}$ and TIR-1 26,27 (Supplementary Data 1d). TIR-1 (Toll/ Interleukin-1 Receptor domain protein), the C. elegans ortholog of SARM1 (Sterile alpha and TIR motif containing protein), functions upstream of the p38 MAPK pathway ${ }^{51}$ to upregulate expression of anti-microbial peptides, including the ShK-like toxin T24B8.5 in the intestine $^{52,53}$ and immune responses to $P$. aeruginosa ${ }^{27}$. Like tir-1 

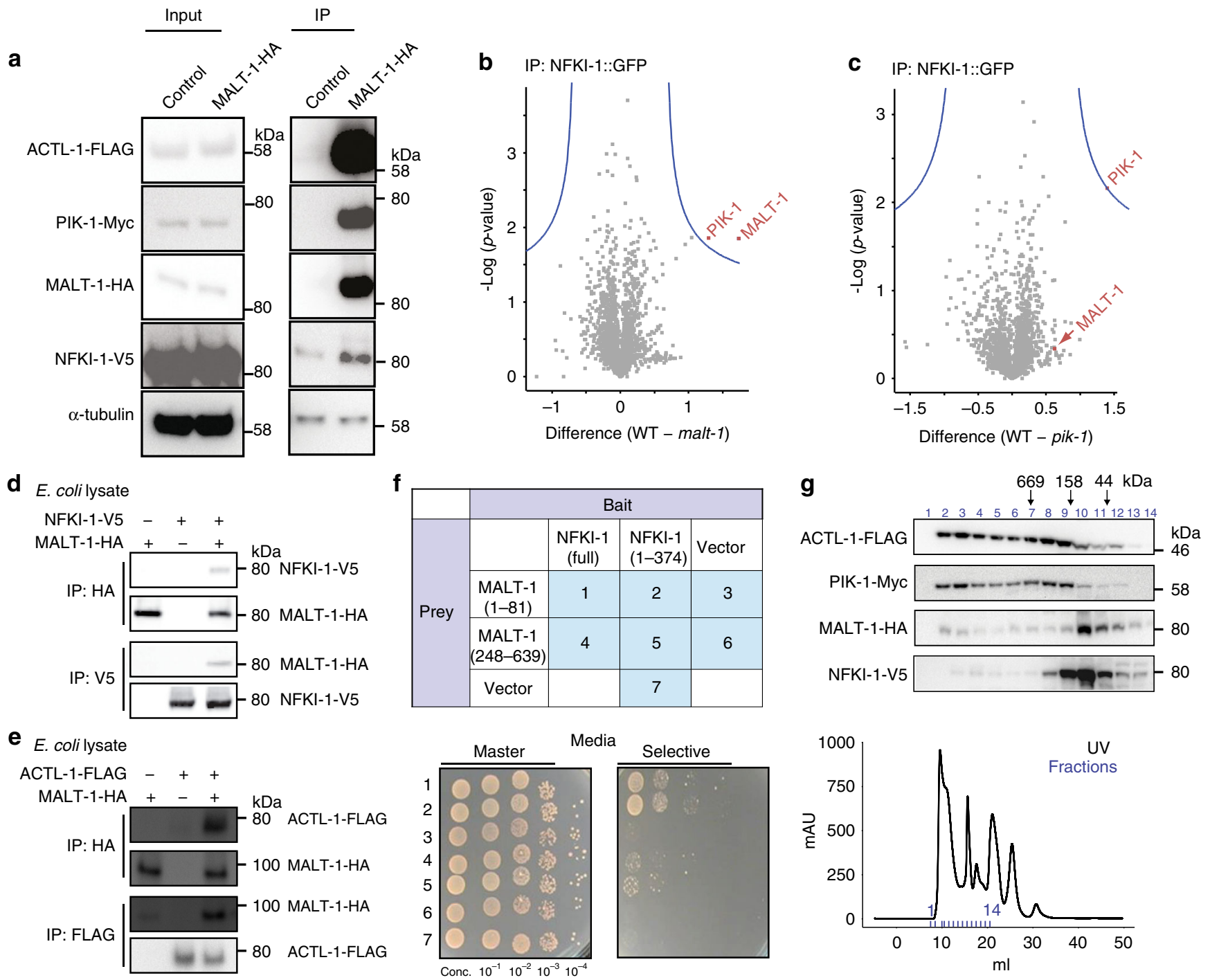

Fig. 6 MALT-1 has scaffolding roles in IL-17 signaling. a Endogenous ACTL-1, PIK-1 and NFKI-1 co-IP with endogenous MALT-1 in npr-1 animals. Anti-HA antibody was used to immunoprecipitate MALT-1 complexes. Half of the lysate was immunoprecipitated with anti-lgG as a control. Tags were knocked in by CRISPR. Similar results were obtained in 3 experiments. b and $\mathbf{c}$ Volcano plot showing quantitative LC-MS/MS of proteins that interact with NFKI-1::GFP in malt-1 and pik-1 mutants compared to wild type. NFKI-1::GFP was purified using GFP-Trap beads, and immunoprecipitated proteins labeled using tandem mass tags (TMT-labeling). The average relative abundance in two biological replicates is shown. $p$-values are reported by a two sample $t$-test. The amount of PIK-1 that co-IPs with overexpressed NFKI-1::GFP is significantly reduced in malt-1(db1194) mutants (b). The relative amount of MALT-1 that co-IPs with NFKI-1 is not significantly decreased in pik-1(tm2167) mutants (c). Peptides derived from MALT-1 and PIK-1 are shown in Supplementary Data 2. d and e IPs of His10-tagged C. elegans ACTL-1-FLAG, MALT-1-HA, and NFKI-1-V5 recombinantly expressed in E. coli show that MALT-1 can directly bind NFKI-1 (d) and ACTL-1 (e). d was performed once, e was performed three times with similar results. $\mathbf{f}$ Interaction of the MALT-1 Death Domain (1-81) with the Nterminus of NFKI-1 (1-374) in a yeast two-hybrid assay using nutritional selection (ADE2). Rows show 10-fold serial dilutions of each of the seven Prey-Bait combination strains tested and shown top. Similar results were obtained in 2 experiments. $\mathbf{g}$ Elution profiles of ACTL-1, PIK-1, MALT-1, and NFKI-1 proteins in a C. elegans extract run on a Superose 6 Gel Filtration column and visualized by immunoblot. All four proteins can be found in high molecular weight complexes. Similar profiles were observed in two runs. See also Supplementary Fig. 7 and Supplementary Data 2.

mutants ${ }^{53}$, malt-1 and ilcr-1 mutants showed reduced T24B8.5 expression (Supplementary Fig. 9a), and this reduction could be rescued by either intestine-specific or nervous system-specific expression of malt-1 (Supplementary Fig. 9b). However, PA14 resistance was reduced in malt-1; tir-1 double mutants compared to malt-1 (Fig. 8i). Thus TIR-1/SARM can still promote PA14 resistance in malt-1 mutants, and while overall IL-17 signaling inhibits the C. elegans immune response to PA14, this effect may reflect the net outcome of opposing influences.

In summary, our data suggest that IL-17 signals through a MALT-1 signalosome to modify neural properties and remodel the behavior and physiology of C. elegans (Fig. 9).

\section{Discussion}

Our data suggest that MALT1 modulates neural circuit function in C. elegans, by acting as a protease and a scaffold. MALT-1 participates in an ACTL-1-IRAK-MALT-1 signaling complex that mediates IL-17 signaling. The high molecular weight of this complex in C. elegans extracts suggests it may form a structure related to the MYD88-IRAK4-IRAK2 Myddosome ${ }^{41}$ and CARMA1-BCL10MALT1 CBM signalosome ${ }^{42}$, although this hypothesis needs further study. MALT-1 directly binds ACTL-1 in vitro, and yeast two hybrid data suggest ACTL-1 directly binds C. elegans IRAK ${ }^{54}$. MALT-1 also interacts directly with NFKI-1, a homolog of mammalian ІкB $\zeta / \mathrm{I} \kappa \mathrm{BNS}$, and can signal through both NFKI-1-dependent 
a

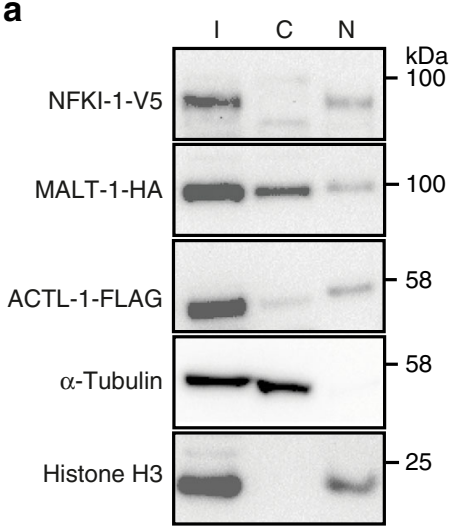

b

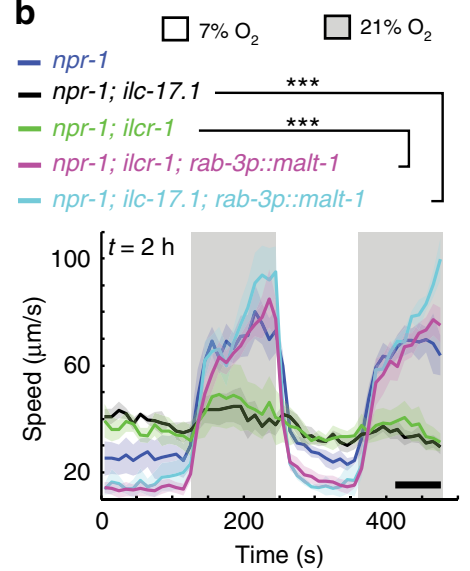

C
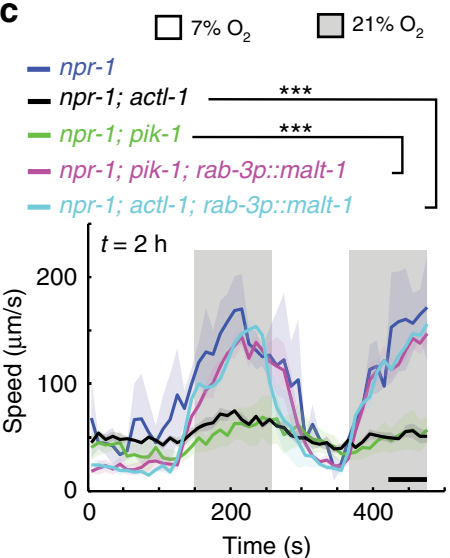

d

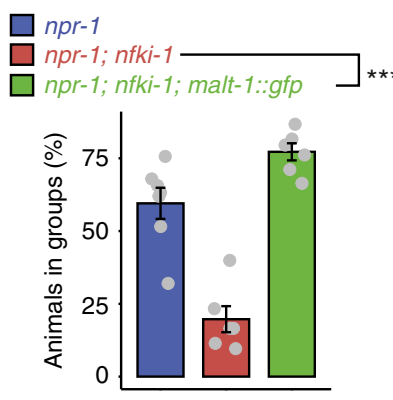

f
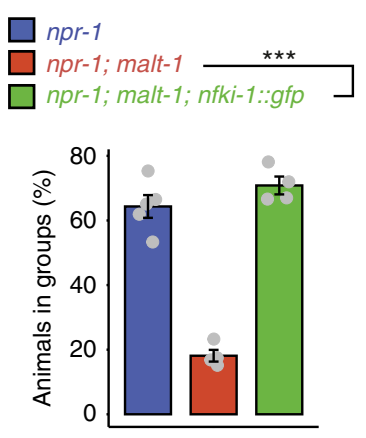

e

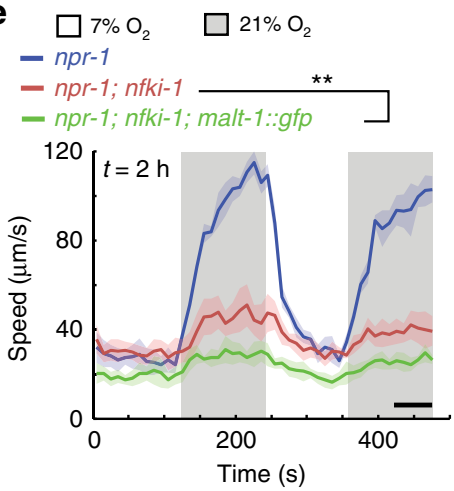

g
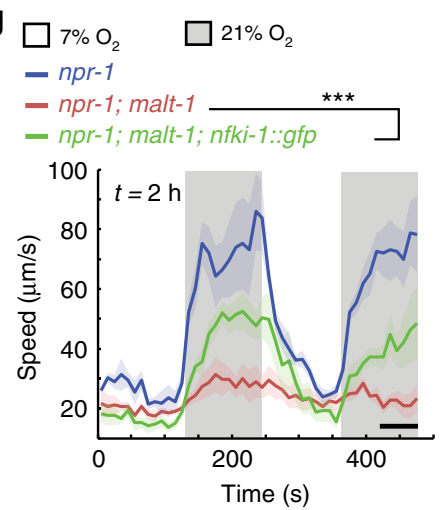

Fig. 7 MALT-1 and NFKI-1 provide partially parallel outputs of IL-17 signaling. a Immunoblot analysis of IL-17 signaling components from nuclear and cytoplasmic fractions of $\mathrm{C}$. elegans lysate. I, input, C, cytosolic, N, nuclear. NFKI-1 is predominately nuclear; ACTL-1 and MALT-1 are distributed between the nucleus and cytoplasm. Similar results were obtained in 5 experiments. $\mathbf{b}$ and $\mathbf{c}$ Overexpressing malt- 1 in neurons, using the rab-3 promoter, restores the arousal response to $21 \% \mathrm{O}_{2}$ to $i / c-17.1$ and ilcr-1 mutants $(\mathbf{b})$, and actl-1 and pik-1 mutants (c). b $n=52$ animals $(n p r-1), n=104$ animals $(n p r-1 ; i l c r-1), n=71$ animals (npr-1; ilcr-1; rab-3p::malt-1), $n=86$ animals ( $n p r-1 ;$ ilc-17.1), $n=61$ animals ( $n$ pr-1; ilc-17.1; rab-3p::malt-1). c $n=19$ animals (npr-1), $n=46$ animals (npr-1; actl-1), $n=26$ animals ( $n$ rr-1; actl-1; rab-3p::malt-1), $n=33$ animals ( $n p r-1 ;$ pik-1), $n=28$ animals ( $n p r-1 ;$ pik-1; rab-3p::malt-1). Plots show average speed (line) and SEM (shaded regions). ${ }^{\star \star \star} P<0.001$, two-sided Mann-Whitney $U$ test. $\mathbf{d}$ and $\mathbf{e}$ Overexpressing malt-1 gDNA also rescues the aggregation phenotype (d), but not the arousal defect (e) of nfki-1 mutants. d $N=7$ assays (npr-1), $N=6$ assays (npr-1; nfki-1 and npr-1; nfki-1; malt-1::gfp). ${ }^{\star \star \star} P=3.5 \mathrm{e}$ -05, one-way ANOVA with Tukey's post hoc HSD. e $n=47$ animals ( $n p r-1), n=79$ animals (npr-1; nfki-1), $n=39$ animals ( $n p r-1 ; n f k i-1 ;$ malt-1::gfp). ${ }^{\star \star} P=$ 0.0067 , two-sided Mann-Whitney $U$ test. $\mathbf{f}$ and $\mathbf{g}$ The aggregation phenotype of malt- 1 is rescued by overexpressing nfki-1 cDNA (f), while speed defects are partially rescued $(\mathbf{g}) . \mathbf{f} N=5$ assays (npr-1), $N=4$ assays (npr-1; malt- 1 and npr-1; malt-1; nfki-1::gfp). ${ }^{\star \star \star} P=7 \mathrm{e}-07$, one-way ANOVA with Tukey's post hoc HSD. $\mathbf{g} n=36$ animals (npr-1), $n=50$ animals (npr-1; malt-1), $n=44$ animals (npr-1; malt-1; nfki-1::gfp). ${ }^{\star \star \star} P=7 \mathrm{e}-07$, one-way ANOVA with Tukey's post hoc HSD. ${ }^{\star \star \star} P=4.7 \mathrm{e}-4$, two-sided Mann-Whitney $U$ test.

and independent mechanisms to alter neuron function and change behavior. The ACTL-1-IRAK-MALT-1-NFKI-1 pathway is present in most neurons of the C. elegans nervous system, and appears to be a neuromodulatory axis impacting multiple phenotypes.

Like ILCR-1 and ILCR-2 ${ }^{17}$, MALT-1 functions in both URX $\mathrm{O}_{2}$ sensors and RMG interneurons to promote escape from $21 \%$
$\mathrm{O}_{2}$. In RMG, ILC-17.1/MALT-1 signaling potentiates $\mathrm{Ca}^{2+}$ responses to pre-synaptic input from $\mathrm{URX} \mathrm{O}_{2}$ sensors, which are tonically activated by $21 \% \mathrm{O}_{2}$. In URX, ILC-17.1/MALT-1 signaling does not appear to disrupt $\mathrm{O}_{2}$-evoked $\mathrm{Ca}^{2+}$ responses, suggesting that it potentiates behavioral arousal to $21 \% \mathrm{O}_{2}$ by augmenting synaptic or gap junctional communication. These 
a

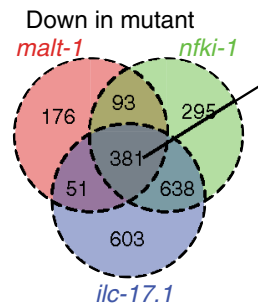

b Up in mutant
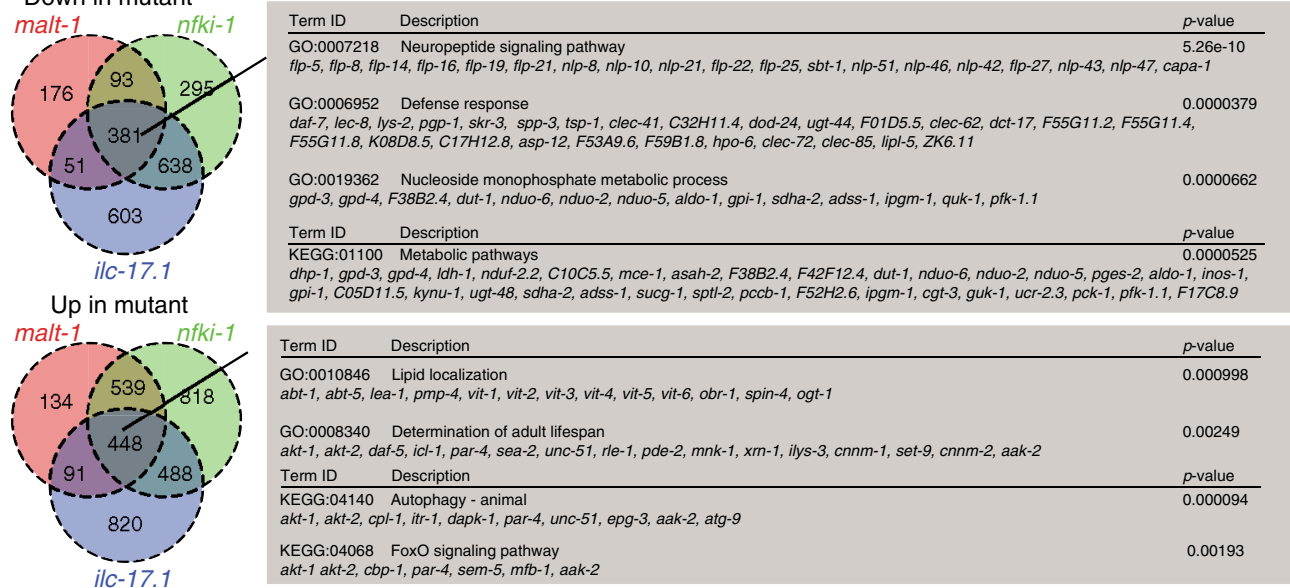

C

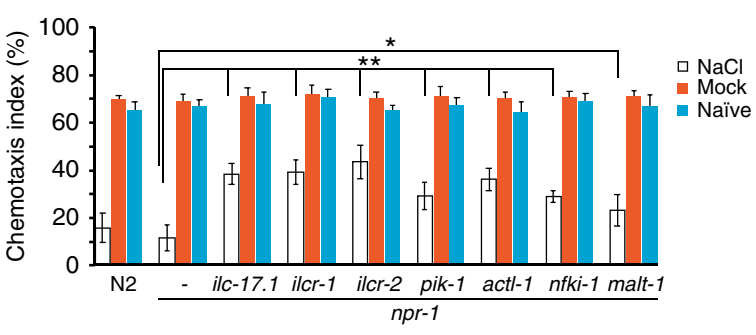

d

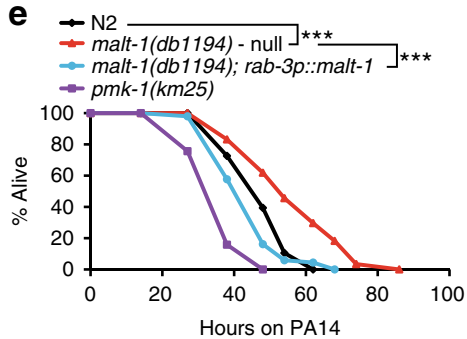

f
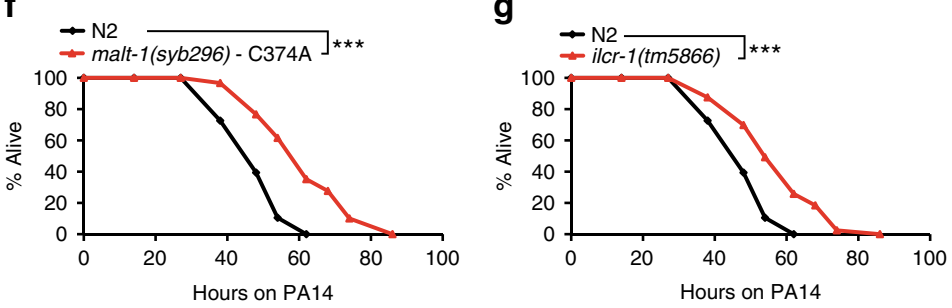

h
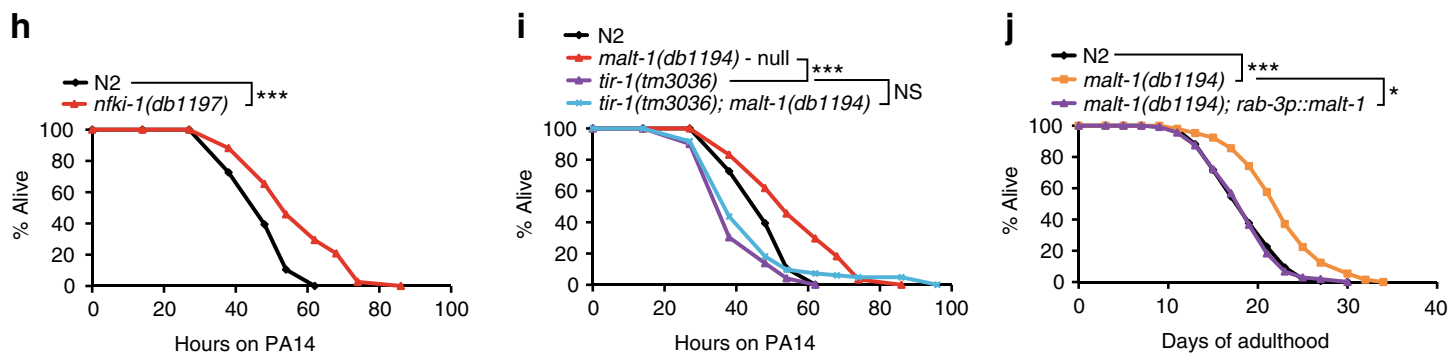

k

I $\rightarrow \mathrm{N} 2$

$=$ malt-1(db1194)

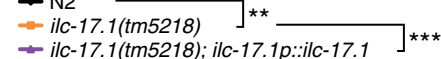

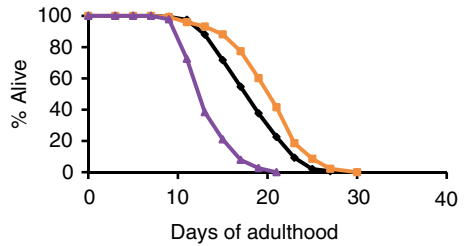

- ilc-17.1 (tm5218)

- ilc-17.1 (tm5218)
- malt-1(db1194); ilc-17.1 (tm5218) $]$ NS

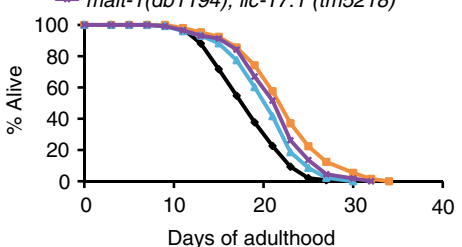

$\mathrm{m} \rightarrow \mathrm{N} 2$

\section{- malt-1(db1194)}

- ilc-17(tm5218); pilc-17.1::ilc-17.1 - malt-1(db1194); pilc-17.1::ilc-17.1 $\left.\right|^{\star * *}$

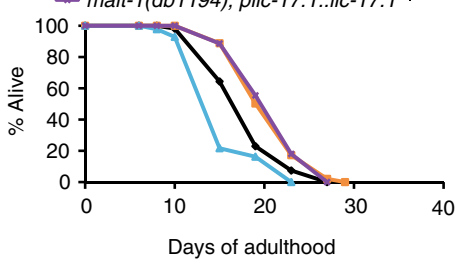

different effects of IL-17 signaling may be indicative of cell-type specific effects on gene expression. Our IP/MS experiments identified transcription factors, chromatin remodeling factors and RNA binding proteins as specific interactors of NFKI-1 and/or MALT-1, but further work is needed to identify cell types in which these interactions are functionally relevant.
Neuronal MALT-1 signaling also modulates pathogen susceptibility and longevity. The nervous system plays an important and conserved role in regulating immunity ${ }^{55-59}$, and multiple neurons ${ }^{60-62}$ and secreted factors ${ }^{63,64}$ that regulate innate immune gene expression in non-neuronal tissues have been discovered. The nervous system also mediates behavioral avoidance of pathogens, by 
Fig. 8 MALT-1 acts downstream of IL-17 signaling to reprogram behavior and physiology. a and b Downregulated (a) and upregulated (b) genes in whole animal RNA-seq profiles of malt-1; npr-1, ilc-17.1; npr-1 and nfki-1; npr-1 double mutants compared to npr-1 controls. Gene ontology (GO) terms and KEGG pathways significantly overrepresented among genes dysregulated in all three mutant conditions are shown $(q$-value $<0.05$, with a minimum log2 (fold-change) of \pm 0.25 ). $\mathbf{c}$ and $\mathbf{d}$ Salt chemotaxis after conditioning by food-withdrawal in the absence or presence of $\mathrm{NaCl}$. ${ }^{\star} P<0.05,{ }^{\star \star} P<0.01,{ }^{\star \star \star} P<$ 0.001 , one-way ANOVA with Tukey's post hoc HSD, $N=6$ assays. d The salt chemotaxis learning defect of ilcr-1 mutants is rescued by driving ilcr-1 expression in many neurons ( $r a b-3$ or flp-21 promoters), or specifically in ASE (flp-6 promoter). e-i PA14 big lawn assays. $n \geq 81$ animals. ${ }^{\star \star \star} P<0.001$, twosided logrank test; precise $n$ numbers and $P$ values are provided in Supplementary Table 1. Mutants lacking malt- 1 (e) or encoding protease-dead malt-1 (f), or defective in other IL-17 signaling components $(\mathbf{g}, \mathbf{h})$ are resistant to P. aeruginosa PA14 in big lawn assays, where animals cannot escape from the PA14 lawn. The enhanced survival of malt-1 mutants is rescued by pan-neuronal expression of malt-1 gDNA. $n \geq 81$ animals. $\mathbf{i}$ The enhanced resistance of malt-1 mutants to PA14 requires TIR-1. Like tir-1 mutants, malt-1; tir-1 double mutants are hypersensitive to PA14 infection. $\mathbf{j}-\mathbf{m}$ Lifespan. $n \geq 92$ animals. ${ }^{\star \star} P<0.01$, ${ }^{\star \star \star} P<0.001$, two-sided logrank test; precise $\mathrm{n}$ numbers and $P$ values are provided in Supplementary Table 3. $\mathbf{j}$ and $\mathbf{k}$ The lifespan of malt-1 and ilc-17.1 mutants is increased compared to N2 controls. The malt-1 phenotype is rescued by expressing malt-1 gDNA pan-neuronally (j) and the ilc-17.1 phenotype can be rescued by expressing ilc-17.1 cDNA from its endogenous promoter (k). I The lifespan phenotypes of malt-1 and ilc-17.1 mutants are not additive. m The shortened lifespan of animals overexpressing ILC-17.1 is abolished in malt-1 mutants. See also Supplementary Fig. 7, Supplementary Tables 1-3 and Supplementary Data 3-6.

a

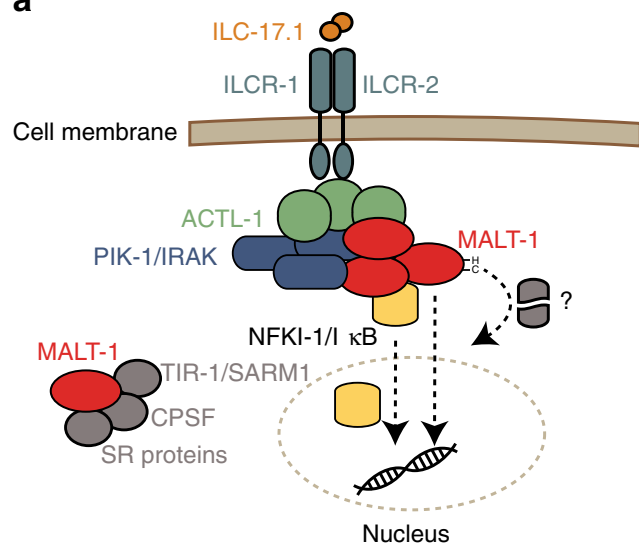

b

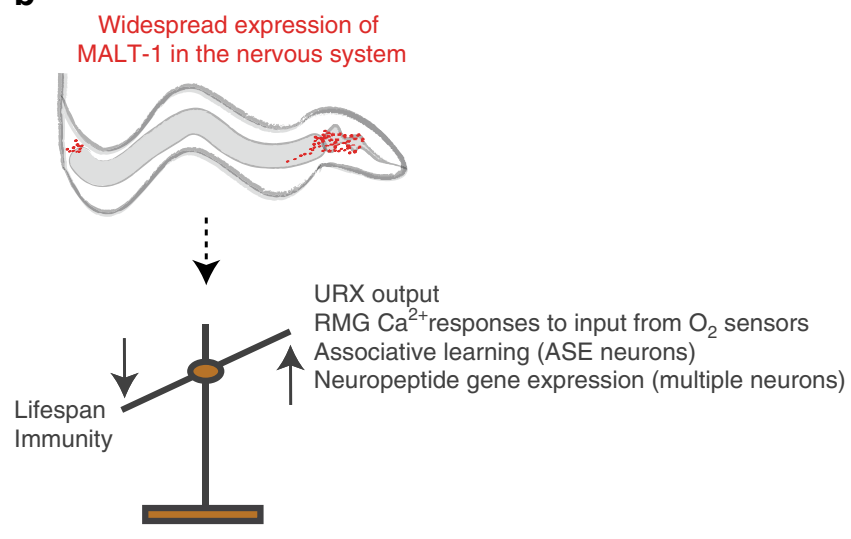

Fig. 9 Model. a Activation of nematode IL-17Rs ILCR-1 and ILCR-2 engages ACTL-1, the C. elegans ACT1-like adapter, probably via their SEFIR domains. ACTL-1 recruits the $C$. elegans IRAK and MALT1 homologs to form the ACT1-IRAK-MALT1 signalosome in the cytoplasm. This serves a scaffolding function to recruit IKBC/NFKI-1, and modulate its actvity by an unknown mechanism. NFKI-1 probably orchestrates changes in the transcriptome of RMG and other cells. MALT1-mediated cleavage of unknown substrate(s) positively regulates NFKI-1 signaling. In parallel to this pathway, MALT-1 forms a complex of unknown function with TIR-1/SARM1, and with multiple RNA-binding proteins. b ILCR receptors and downstream signaling components including MALT-1 are expressed in many neurons. This neuronal signaling cassette alters associative learning, as well as $\mathrm{O}_{2}$-escape behaviors, and suppresses lifespan and immunity.

mechanisms that can be innate or learned ${ }^{49,65}$. Our data suggest that neuronal ILC-17.1/MALT-1 signaling reduces survival on Pseudomonas aeruginosa by non-behavioral mechanisms. A simple model is that by altering neural circuit activity ILC-17.1 can change immune gene expression, for example in the intestine.

MALT1-like paracaspases are found in organisms lacking other CBM components ${ }^{18}$, suggesting MALT1 has unknown functions that predate its coaction with Bcl10 and CARD domain proteins. Our results raise the possibility that one ancestral function was in IL-17 signaling. As IL-17Rs are found throughout metazoa ${ }^{66}$, we speculate that the ACTL-1-IRAK-MALT-1 complex we have identified is the original and primary mechanism by which IL17Rs signal in non-amniote animals, from cnidarians to cephalochordates. In amniotes, ACT1 orthologs have lost a death domain (DD) that is present in ACT1 orthologs from most other lineages ${ }^{66}$. DDs mediate homotypic interactions in large immune complexes such as the Myddosome ${ }^{67}$, and are present in both MALT1 and IRAKs. The DD-SEFIR domain architecture of ACT1 present in non-amniotes resembles the DD-TIR domain structure of MyD88, since TIR and SEFIR domains are related ${ }^{68}$. Interestingly, proximity labeling studies find MALT1 associates with MyD88 in DLBCL cells ${ }^{69}$, although the functional consequences of this interaction are not yet known. Recent studies have also speculated that mammalian MALT1 is recruited to IL-
17 signaling complexes ${ }^{70,71}$. Direct evidence for this is lacking, but if correct, this would mirror our results in C. elegans.

One area of future study will investigate how MALT-1 alters neural function. Although we find that MALT-1 protease activity is essential, we have not identified its neural substrate(s). Substrates of mammalian MALT1 with orthologs in C. elegans include the RNA binding proteins roquin-1/2 (RLE-1), which target RNAs for degradation, the endoribonuclease regnase-1 (REGE-1), and the CYLD (CYLD-1) deubiquitinase ${ }^{72-74}$. We did not detect these proteins in our proteomic analyses (Supplementary Data 1d). However, our IP/MS data indicate that besides binding NFKI-1, MALT-1 interacts with multiple RNA binding proteins, including splicing and polyadenylation factors, and with the C. elegans ortholog of SARM1, called TIR-1. TIR-1 regulates C. elegans gustatory and olfactory plasticity ${ }^{29}$, and proteostasis $^{75}$, by modulating MAPK pathways, making it a plausible target for regulation by MALT-1.

The closest mammalian homolog of NFKI-1, ІкB $\zeta$, is a nuclearlocalized protein that acts as a transcriptional regulator, and is rapidly induced by inflammatory stimuli, including IL-17. IкB $\zeta$ is thought to mediate its effects on gene expression primarily by regulating chromatin structure, although how it is recruited to target genes is not completely understood since it lacks a DNA binding domain ${ }^{76,77}$. Our IP/MS data find NFKI-1 physically 
interacts with the CREB binding protein (CREBBP), $c b p-1$, which is a histone acetyltransferase, consistent with NFKI-1 acting to modify chromatin structure. Most of the gene expression changes highlighted by our RNA-seq studies likely reflect secondary consequences of IL-17 signaling defects, although some genes may be directly regulated by NFKI-1.

Our biochemical and genetic analyses of IL-17 signaling in C. elegans have identified functional roles and biochemical interactions previously undescribed in mammals. An outstanding challenge is to examine which of these are conserved in mammals. Does MALT1 play a role in modulating mammalian neural function, given that neurons can express receptors with an ITAM (immunoreceptor tyrosine-based activation motif) ${ }^{19-21}$, as well as GPCRs, that in immune cells signal through MALT1? Does MALT1 contribute to known neuronal responses to IL-17 13,14,78 ?

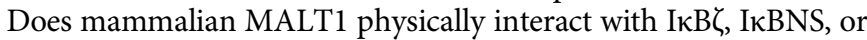
SARM1? In summary, we have used an invertebrate model system, with the relative simplicity this offers, and its advantages for genetics, biochemistry and single neuron analysis, to probe how key immune molecules signal in neurons to alter circuit function.

\section{Methods}

Strains and genetics. C. elegans were maintained on nematode growth medium (NGM) at room temperature $\left(22^{\circ} \mathrm{C}\right)$ with E. coli OP50 food. Strains used are provided in Supplementary Table 5.

Whole genome sequencing showed that the aggregation-defective AX3621 strain was defective in malt-1. We used SNP mapping ${ }^{79}$ to investigate if the aggregation defect was linked to malt-1. We crossed AX3621 animals with the AX288 [lon-2(e678) npr-1(ad609)] strain; AX288 was constructed by backcrossing lon-2 npr-1(ad609) X 16× into the CB4856 (Hawaiian) wild strain. The npr-1 (ad609) allele confers stronger aggregation than the CB4856 Hawaiian strain routinely used for mapping. We 'singled' F2 animals, and scored their progeny for aggregation. Animals from non-aggregating F3 lines were pooled, and their DNA extracted and sequenced. Sequencing libraries were made using the Nextera DNA Library kit, and sequenced on a HiSeq 2500 (Illumina) machine with $125 \mathrm{bp}$ paired-end reads. Sequencing data were analyzed using CloudMap ${ }^{79}$.

Molecular biology. Primers used in this study are provided in Supplementary Table 6. C. elegans expression constructs were generated using MultiSite Gateway Recombination (Invitrogen). To amplify the malt-1 promoter $(4 \mathrm{~kb})$ we used primers ggggACAACTTTGTATAGAAAAGTTGctgc cggtggattccaacatattg and ggggACTGCTTTTTTGTACAAACTTGtctgaaattggggttcaagaaatttatttttgatttttaaaata to amplify the malt-1 ORF (gDNA):

ggggACAAGTTTGTACAAAAAAGCAGGCTtttcagaaaaatgaacacaaacttggcggagt tc and ggggACCACTTTGTACAAGAAAGCTGGGTActgtagacatttgattcttgtaatcaa aatatgaccaatatc

and to amplify malt-1 cDNA:

ggggACAAGTTTGTACAAAAAAGCAGGCTtttcagaaaaatgaacacaaacttggcggagt tc and ggggACCACTTTGTACAAGAAAGCTGGGTATTACTGTAGACATTTGA TTC TTGTAATCAAAATATGACCAATATCAACATTC.

The Q5 Site-Directed Mutagenesis Kit (NEB) was used to create malt-1(C374A) cDNA, with the following primers:

TCTTGATGTCgcCAGAAAATTTGTTCCATATG and gcgcgtcaagttgtGCCTGAC GACGAGTTGTGCTGTTTTAGAGCTAGAA

To generate deletions in the malt-1 locus by CRISPR/Cas9 we expressed a gaucagguauccaccguag short guide from the $r p r-1$ promoter ${ }^{80}$. The primers used to amplify this sequence for insertion into an EcoRI-cut expression plasmid (addgene \#48961) were gcgcgtcaagttgtGgatcaggtatccaccgtagGTTTTAGAGCTAGAA and TTCTAGCTCTAAAACctacggtggatacctgatcCacaacttgacgcgc.

Expression constructs were injected at $50 \mathrm{ng} / \mu \mathrm{l}$, with the exception of CRISPRCas9 mixes that were prepared as previously described ${ }^{80}: 30 \mathrm{ng} \mathrm{ng} / \mu \mathrm{l}$ eft-3::cas 9 , $100 \mathrm{ng} / \mu \mathrm{l}$ sgRNA, $30 \mathrm{ng} \mathrm{ng} / \mu \mathrm{l} \mathrm{cc}: \mathrm{GFP}$.

The following alleles were generated by SunyBiotech (Fuzhou, China) using CRISPR/Cas9-based genome editing: malt-1(syb296), actl-1(syb412), pik-1(syb378), malt-1(syb573), and nfki-1(syb617). We verified modified sequences using Sanger sequencing (Supplementary Table 4).

Behavioral assays. Behavioral assays were performed at room temperature $\left(22^{\circ} \mathrm{C}\right)$ Aggregation was assayed as previously described ${ }^{48} ; 60$ young adults were picked onto a plate seeded with $100 \mu \mathrm{l}$ OP50 $48 \mathrm{~h}$ previously. Animals were left undisturbed for $2 \mathrm{~h}$ and then scored blind to genotype. The \% of animals in groups was calculated, with a group defined as 3 or more animals in contact with one another. Statistical comparisons were made using ANOVA (with RStudio (v 1.0.143)).

Locomotory responses to $\mathrm{O}_{2}$ stimuli were measured as described previously 31,81 with minor modifications. 15-25 young adults were picked onto a plate seeded with
$20 \mu \mathrm{l}$ OP50 14-18 h previously, and covered with a microfluidic PDMS chamber. Defined $\mathrm{O}_{2}$ mixtures (balance nitrogen) were bubbled through $\mathrm{H}_{2} \mathrm{O}$ and delivered to the PDMS chamber at a rate of $1.4 \mathrm{ml} / \mathrm{min}$ using a PHD 2000 Infusion syringe pump (Harvard Apparatus). Video recordings were acquired at $2 \mathrm{fps}$ with FlyCapture 1.X software (FLIR Systems), using a Point Gray Grasshopper camera mounted on a Leica MZ6 microscope. Speed and reversals were measured using Zentracker custom software (https://github.com/wormtracker/zentracker). To measure phenotypes associated with IL-17 signaling defects, worms were left undisturbed for $2 \mathrm{~h}$ on assay plates prior to recording.

To measure thrashing, single animals were placed into individual wells containing $50 \mu \mathrm{l} \mathrm{M} 9$ buffer. The number of complete body bends per minute was measured by a scorer blind to genotype.

Heat-shock. As reported previously, the $h s p-16.41$ heat shock promoter is leaky in animals grown at room temperature. We therefore kept animals at $15^{\circ} \mathrm{C}$ until the time of heatshock (late L4). To induce heat-shock, parafilm-wrapped plates were submerged in a $34^{\circ} \mathrm{C}$ water bath for $30 \mathrm{~min}$, and then recovered at room temperature until the time of assay.

Light microscopy. Worms were immobilized with $25 \mathrm{mM}$ sodium azide on $2 \%$ agarose pads. $Z$ stacks from animals expressing MALT-1::GFP and MALT-1::RFP were acquired on an Inverted Leica SP8 confocal microscope using a $\times 63 / 1.20$ water objective, using the LAS X software platform (Leica). Figure panels were obtained using the Z-project (average intensity) function in FIJI (ImageJ v2.0.0-rc-69).

We quantified GFP intensity in L4 animals expressing the agIs219(pT24B8.5:: GFP) transgene ${ }^{53}$ using NIS-elements (Nikon) and a Nikon Ti2 microscope with a Niji LED light source (Bluebox Optics, Huntingdon, UK) and a NEO scientific CMOS camera (Andor, Belfast, UK), with a $\times 10$ objective (Nikon, Tokyo, Japan) and $50 \mathrm{~ms}$ exposure time.

Calcium imaging. Animals expressing cameleon YC2.60 were imaged with a $\times 2$ AZ-Plan Fluor objective (Nikon) on a Nikon AZ100 microscope fitted with ORCAFlash4.0 digital cameras (Hamamatsu). Excitation light was provided from an Intensilight C-HGFI (Nikon), through a 438/24 nm filter and an FF458DiO2 dichroic (Semrock). Emission light was split using a TwinCam dual camera adapter (Cairn Research) and passed through CFP (483/32 nm) and YFP (542/27) filters, and a DC/T510LPXRXTUf2 dichroic. We acquired movies using NIS-Elements (Nikon), with $100 \mathrm{~ms}$ exposure time.

To image neural activity in freely moving animals (Supplementary Fig. 4g) single young adults were transferred to peptone-free agar plates spotted with $4 \mu \mathrm{l}$ of concentrated OP50 food in M9 buffer, and imaged at $2 \times$ zoom. For all other figures, 4-8 young adults were transferred to peptone-free agar plates, immobilized on a $2 \mu \mathrm{l}$ patch of concentrated OP50 in M9 buffer using Dermabond adhesive, leaving the nose exposed, and imaged at $4 \times$ zoom.

Immunoprecipitation from $\boldsymbol{C}$. elegans. For co-IP experiments analyzed by LCMS/MS, C. elegans lysis and affinity purification was performed as previously described $^{82}$ with minor modification. Lysis buffer A was prepared with $50 \mathrm{mM}$ HEPES (pH 7.4), $1 \mathrm{mM}$ EGTA, $1 \mathrm{mM} \mathrm{MgCl}_{2}, 100 \mathrm{mM} \mathrm{KCl,} 10 \%$ glycerol, $0.05 \%$ NP40, $1 \mathrm{mM}$ DTT, 0.1 M PMSF and 1 complete EDTA-free proteinase inhibitor cocktail tablet (Roche Applied Science) per $12 \mathrm{ml}$. Unsynchronized worms grown in liquid were washed twice in M9 and once in ice-cold lysis buffer A, then snapfrozen by dropwise addition to $\mathrm{LN}_{2}$ in preparation for cryogenic grinding. Worm popcorn was pulverized using a Freezer/Mill (SPEX SamplePrep). Crude extract was clarified at $4{ }^{\circ} \mathrm{C}$ for $10 \mathrm{~min}$ at $20,000 \times g$, and again for $20 \mathrm{~min}$ at $100,000 \times g$ with a TLA-100 rotor (Beckman Coulter). For IP, roughly equal volumes of sample and control lysate were incubated with $100 \mu$ l GFP-Trap MA (ChromoTek gtma), MycTrap MA (ChromoTek ytma), or anti-FLAG M2 magnetic beads (Sigma M8823) for $3-4 \mathrm{~h}$ at $4{ }^{\circ} \mathrm{C}$, then washed twice with $50 \mathrm{mM}$ HEPES, $100 \mathrm{mM} \mathrm{KCl}$. Purified complexes were eluted in SDS-sample buffer at $95^{\circ} \mathrm{C}$ and fractionated by SDSPAGE prior to characterization by LC-MS/MS.

For co-IP experiments analyzed by Western blot, the following modifications were made. Lysis buffer B contained $50 \mathrm{mM}$ HEPES (pH 7.4), $100 \mathrm{mM} \mathrm{KCl,} \mathrm{0.05 \%}$ NP40, $1 \mathrm{mM}$ DTT, 0.1 M PMSF and 1 complete EDTA-free proteinase inhibitor cocktail tablet (Roche Applied Science) per $12 \mathrm{ml}$. Crude extract was clarified at $4{ }^{\circ} \mathrm{C}$ for $30 \mathrm{~min}$ at $18,000 \times g$. For immunoprecipitation, half of the lysate was incubated with anti-HA agarose (Sigma A2095) for $30 \mathrm{~min}$ at $4{ }^{\circ} \mathrm{C}$, then washed $3 \times$ with $50 \mathrm{mM}$ HEPES, $100 \mathrm{mM} \mathrm{KCl}$. As a control, the other half of the lysate was incubated with IgG-agarose (Sigma A0919).

Identification of protein-protein interactions by MS. Gel samples were destained with $50 \% \mathrm{v} / \mathrm{v}$ acetonitrile and $50 \mathrm{mM}$ ammonium bicarbonate, reduced with $10 \mathrm{mM}$ DTT, and alkylated with $55 \mathrm{mM}$ iodoacetamide. Proteins were digested with $6 \mathrm{ng} / \mu \mathrm{l}$ trypsin (Promega, UK) overnight at $37^{\circ} \mathrm{C}$, and peptides extracted in $2 \% \mathrm{v} / \mathrm{v}$ formic acid $2 \% \mathrm{v} / \mathrm{v}$ acetonitrile, and analyzed by nano-scale capillary LCMS/MS (Ultimate U3000 HPLC, Thermo Scientific Dionex) at a flow of $\sim 300 \mathrm{~nL} /$ min. A C18 Acclaim PepMap100 $5 \mu \mathrm{m}, 100 \mu \mathrm{m} \times 20 \mathrm{~mm}$ nanoViper (Thermo Scientific Dionex), trapped the peptides prior to separation on a C18 Acclaim PepMap100 $3 \mu \mathrm{m}, 75 \mu \mathrm{m} \times 250 \mathrm{~mm}$ nanoViper. Peptides were eluted with an 
acetonitrile gradient. The analytical column outlet was interfaced via a nano-flow electrospray ionization source with a linear ion trap mass spectrometer (Orbitrap Velos, Thermo Scientific). Data dependent analysis was performed using a resolution of 30,000 for the full MS spectrum, followed by ten MS/MS spectra in the linear ion trap. MS spectra were collected over a $\mathrm{m} / \mathrm{z}$ range of 300-2000. MS/MS scans were collected using a threshold energy of 35 for collision-induced dissociation. LC-MS/MS data were searched against the UniProt KB database using Mascot (Matrix Science), with a precursor tolerance of $10 \mathrm{ppm}$ and a fragment ion mass tolerance of $0.8 \mathrm{Da}$. Two missed enzyme cleavages and variable modifications for oxidized methionine, carbamidomethyl cysteine, pyroglutamic acid, phosphorylated serine, threonine and tyrosine were included. MS/MS data were validated using the Scaffold program (v4.10.0, Proteome Software Inc).

Quantification of NFKI-1 interacting peptides by TMT labeling. Protein samples on beads were reduced with $10 \mathrm{mM}$ DTT at $56^{\circ} \mathrm{C}$ for $30 \mathrm{~min}$ and alkylated with 15 $\mathrm{mM}$ iodoacetamide (IAA) in the dark at $22^{\circ} \mathrm{C}$ for $30 \mathrm{~min}$. Alkylation was quenched by adding DTT and the samples digested with trypsin (Promega, $1.25 \mu \mathrm{g}$ ) overnight at $37^{\circ} \mathrm{C}$. After digestion, supernatants were transferred to a fresh Eppendorf tube, the beads were extracted once with $80 \%$ acetonitrile/ $0.1 \%$ TFA and combined with the corresponding supernatant. The peptide mixtures were then partially dried in a Speed Vac and desalted using home-made C18 (3 M Empore) stage tip filled with $4 \mu \mathrm{l}$ of poros R3 (Applied Biosystems) resin. Bound peptides were eluted sequentially with $30 \%, 50 \%$, and $80 \%$ acetonitrile in $0.1 \%$ TFA and lyophilized.

Dried peptide mixtures from each condition were re-suspended in $40 \mu \mathrm{l}$ of $250 \mathrm{mM}$ triethyl ammonium bicarbonate. $0.8 \mathrm{mg}$ of TMT 6plex reagents (Thermo Fisher Scientific) was re-constituted in $41 \mu \mathrm{l}$ anhydrous $\mathrm{MeCN}$. Twenty microliter of TMT reagent was added to each peptide mixture and incubated for $1 \mathrm{~h}$ at r.t. The labeling reactions were terminated by incubation with $4.4 \mu \mathrm{l}$ of $5 \%$ hydroxylamine for $15 \mathrm{~min}$. For each condition, the labeled samples were pooled, Speed Vac to remove acetonitrile, desalted and then fractionated with home-made C18stage tip using $10 \mathrm{mM}$ ammonium bicarbonate and acetonitrile gradients. Eluted fractions were acidified, partially dried down in speed vac and ready for LC-MSMS.

Peptides were separated on an Ultimate 3000 RSLC nano System (Thermo Scientific), using a binary gradient consisting of buffer A ( $2 \% \mathrm{MeCN}, 0.1 \%$ formic acid) and buffer B ( $80 \% \mathrm{MeCN}, 0.1 \%$ formic acid). Eluted peptides were introduced directly via a nanospray ion source into a Q Exactive Plus hybrid quardrupoleOrbitrap mass spectrometer (Thermo Fisher Scientific). The mass spectrometer was operated in standard data dependent mode, performed survey full-scan (MS, $\mathrm{m} / z=$ $350-1600$ ) with a resolution of 140,000 , followed by MS2 acquisitions of the 15 most intense ions with a resolution of 35,000 and NCE of 33\%. MS target values of 3e6 and MS2 target values of 1e5 were used. Dynamic exclusion was enabled for $40 \mathrm{~s}$.

The acquired MSMS raw files were processed using MaxQuant ${ }^{83}$ with the integrated Andromeda search engine (v.1.5.5.1). MSMS spectra were searched against the Caenorhabditis elegans UniProt Fasta database (July 2017).

Carbamidomethylation of cysteines was set as fixed modification, while methionine oxidation and $\mathrm{N}$-terminal acetylation (protein) were set as variable modifications. Protein quantification required 1 (unique + razor) peptide. Other parameters in MaxQuant were set to default values. MaxQuant output file, proteinGroups.txt was then processed with Perseus software (v 1.5.5.0). After uploading the matrix, the data was filtered, to remove identifications from reverse database, modified peptide only, and common contaminants. Each peptide channel was normalized to the median and $\log 2$ transformed.

Protein expression in E. coli. His10-tagged C. elegans MALT-1, ACTL-1, and NFKI-1 were expressed in E. coli strain BL21(DE3) and purified using Ni-NTA agarose (Qiagen) or HisPur Cobalt resin (ThermoFisher Scientific).

Sub-cellular fractionation. Nuclear/cytoplasmic fractionation was performed as described previously ${ }^{84}$. Young adult worms were washed 3-5 times in M9, and twice in hypotonic buffer ( $15 \mathrm{mM}$ HEPES, $10 \mathrm{mM} \mathrm{KCl,} 5 \mathrm{mM} \mathrm{MgCl} 2,0.1 \mathrm{mM}$ EDTA, $350 \mathrm{mM}$ sucrose). Lysis was on ice in complete hypotonic buffer plus $1 \mathrm{mM}$ DTT and 1 complete EDTA-free proteinase inhibitor cocktail tablet (Roche Applied Science) per $12 \mathrm{ml}$, using a motorized pellet pestle (Sigma Z359971, Z359947) until most worm carcasses were homogenized. Worm debris was pelleted at $500 \times g(2 \times$ $5 \mathrm{~min}$ ), and $5 \%$ of the resultant supernatant was kept as the input fraction. Nuclei were pelleted at $4000 \times g(5 \mathrm{~min})$, and the resulting supernatant centrifuged again at $17,000 \times g$ and kept as the cytoplasmic fraction. Nuclear pellets were washed twice in complete hypotonic buffer and dissolved in complete hypertonic buffer $(15 \mathrm{mM}$ HEPES, $400 \mathrm{mM} \mathrm{KCl}, 5 \mathrm{mM} \mathrm{MgCl}_{2}, 0.1 \mathrm{mM}$ EDTA, $0.1 \%$ Tween $20,10 \%$ glycerol, $1 \mathrm{mM}$ DTT, complete EDTA-free proteinase inhibitor as above).

Size-exclusion chromatography. C. elegans lysate was prepared as described above for LC-MS/MS, and loaded onto a Superose 6 Increase 10/300 GL column. The column was equilibrated with Lysis buffer A and $1 \mathrm{ml}$ fractions were collected using Unicorn 7.0 (GE Healthcare Life Sciences).

Immunoblotting. After SDS-PAGE using Bolt 4-12\% Bis-Tris Plus gels (ThermoFisher Scientific), protein was transferred to PVDF membrane (0.45-micron pore size, ThermoFisher Scientific) using the XCell II Blot Module (ThermoFisher
Scientific). Membranes were blocked with $5 \%$ milk for $1 \mathrm{~h}$, then incubated with primary antibody overnight at $4{ }^{\circ} \mathrm{C}$, followed by secondary antibody for $1 \mathrm{~h}$ at RT. Unbound antibody was washed away with PBST $(3 \times 5 \mathrm{~min})$, and SuperSignal West Pico PLUS Chemiluminescent Substrate (ThermoFisher Scientific) used for detection. The following commercially available antibodies were used: anti-FLAG M2-Peroxidase (A8592, Sigma A8592-2MG, diluted 1:1000), anti-Myc (9B11, Cell Signaling \#2276, diluted 1:1000), anti-HA (C29F4, Cell Signaling \#3724, diluted 1:1000), anti-V5 (A190, Bethyl Laboratories A190-120A, diluted 1:1000), antiHistone H3 (Cell Signaling \#9715, diluted 1:1000), anti-alpha tubulin (DM1A, abcam ab40742, diluted 1:4000), anti-Rabbit IgG (Bio-Rad \#1706515, diluted 1:3000), and anti-Mouse IgG (Bio-Rad \#1706516, diluted 1:3000). Western blots were imaged with Image Lab 4.1 (Bio-Rad).

Yeast two-hybrid assays. Yeast two-hybrid assays were performed by Panbionet (http://panbionet.com). cDNAs encoding NFKI-1 (full length) and NFKI-1 (1-374) were amplified by PCR and cloned into pGBKT7 vector, and cDNAs encoding MALT-1 (1-81) and MALT-1 (248-639) were amplified by PCR and cloned into pGADT7 vector (Clontech). Plasmids were transformed into the AH109 yeast strain, which expresses HIS3 and ADE2 reporters. Transformants were dropped separately onto SD-LW, SD-LWA and SD-LWH media containing $10 \mathrm{mM}$ of 3-AT (3-amino-1,2,3-triazole), a competitive inhibitor of the HIS3 protein (His3p).

RNA preparation. 10 Gravid adults were allowed to lay eggs for $2 \mathrm{~h}$ on an OP50 lawn seeded $24 \mathrm{~h}$ previously, before being picked away. 8-10 plates were used per replicate, and all genetic backgrounds were prepared in parallel. Once animals reached late L4 stage, they were washed twice in $\mathrm{M} 9$ and frozen in liquid $\mathrm{N}_{2}$. One milliliter Qiazol Lysis Reagent (Qiagen) and 300-400 $\mu \mathrm{l} 0.7 \mathrm{~mm}$ Zirconia beads (BioSpec) were added to worm pellets for mechanical disruption. Samples were disrupted with a TissueLyser (Qiagen), using $1 \mathrm{~min}$ at maximum power followed by 1 min on ice (repeated 4 times). RNA was extracted using the RNeasy Plus Universal Mini Kit (Qiagen), following the manufacturer's instructions with the exception that 1-Bromo-3-chloropropane (Sigma, B673) was used instead of chloroform.

Libraries were prepared using the TruSeq Stranded mRNA kit (Illumina) with polyA capture for mRNA, and sequenced on a HiSeq 4000 platform (Illumina) with $50 \mathrm{bp}$ single-end reads. We sequenced five independent biological replicates for $n \mathrm{pr}-1$ ilc-17.1, and six for $n p r-1$, malt-1; npr-1, and npr-1 nfki-1. Reads were aligned to the $C$ elegans genome using TopHat v2.1.0, and expression quantified using Cufflinks v2.2.1. For statistical comparisons a $q$-value $<0.05$ was considered significant. Only genes for which the FPKM (Fragments Per Kilobase of transcript per Million mapped reads) was $\geq 1$ in $n p r-1$, and for which the $\log 2$ (fold change) between conditions was $\geq 0.25$ were included in Fig. 8. Genes whose expression oscillates during development ${ }^{85}$ were excluded.

$\mathrm{GO}$ terms for these genes were retrieved and GO enrichment calculated using $\mathrm{g}$ Profiler (version e94_eg41_p11_592d91786). Terms with multiple testing corrected $p$-values $<0.05$ were considered enriched.

Associative learning assay. Chemotaxis assays were performed as previously described ${ }^{87}$ with minor modifications. To establish salt gradients, $100 \mathrm{mM} \mathrm{NaCl}$ agar plugs were left overnight on assay plates containing $1 \mathrm{mM} \mathrm{CaCl}, 1 \mathrm{mM}$ $\mathrm{MgSO}_{4}, 25 \mathrm{mM} \mathrm{K}_{2} \mathrm{HPO}_{4} \mathrm{pH}$ 6. Plugs were removed immediately before the assay, and replaced with $1 \mu \mathrm{l} 1 \mathrm{M} \mathrm{NaN}_{3}$. Another $1 \mu \mathrm{l} \mathrm{NaN}$ was added equidistant from the starting point as a control. The chemotaxis index was calculated as $(\mathrm{A}-\mathrm{B}) / \mathrm{N}$, where $\mathrm{A}$ was the number of animals within $1 \mathrm{~cm}$ of the peak of the salt gradient, $\mathrm{B}$ was the number of animals within $1 \mathrm{~cm}$ of the control spot, and $N$ was the number of all animals. Conditioning was performed as described previously ${ }^{46}$. Synchronized young adults raised on OP50 were washed three times in CTX buffer $(5 \mathrm{mM}$ $\mathrm{K}_{2} \mathrm{HPO}_{4} \mathrm{pH} 6,1 \mathrm{mM} \mathrm{CaCl}$ and $1 \mathrm{mM} \mathrm{MgSO}_{4}$ ), then left for $4 \mathrm{~h}$ on NGM agar with no $\mathrm{NaCl}$ (mock) or $300 \mathrm{mM} \mathrm{NaCl}$ (conditioned). For each transgenic strain the behavior of animals bearing the transgene was compared to that of their nontransgenic siblings.

P. aeruginosa killing assays. Slow killing assays were performed with $10 \mu \mathrm{M} \mathrm{5-}$ fluorodeoxyuridine $(\mathrm{FUdR})^{88}$. Synchronized L4 animals raised on OP50 were added to $0.35 \%$ peptone NGM plates, seeded the day before with PA14. Animals were scored every $12 \mathrm{~h}$, and counted as dead if they did not respond to prodding. Logrank tests with Bonferroni correction were performed using OASIS (On-line Application for Survival Analysis, https://sbi.postech.ac.kr/oasis/ ${ }^{89}$ ).

Lifespan analyses. Lifespan assays were performed on OP50, starting on day 1 of adulthood ${ }^{90}$. Scoring and statistical analyses were performed as described above for $P$. aeruginosa killing assays.

Statistics. Statistical tests and $\mathrm{n}$ values used for experiments in this paper are indicated in the corresponding figure legend or methods section. For salt chemotaxis and aggregation behavior, statistical significance between groups was determined using one-way ANOVA with Tukey's post hoc HSD. Differences in locomotory response and FRET levels (yellow cameleon) were evaluated by MannWhitney $U$ test. Logrank tests with Bonferroni correction were used to compare 
genotypes in lifespan and PA14 survival assays using OASIS (Online Application for Survival Analysis, https://sbi.postech.ac.kr/oasis/ ${ }^{89}$ ).

Reporting summary. Further information on research design is available in the Nature Research Reporting Summary linked to this article.

\section{Data availability}

Data supporting the findings of this study are provided as a Source Data file, and are available from the corresponding author. Full scans of blots are provided in Supplementary Fig. 10. RNA-seq data has been deposited in Gene Expression Omnibus (GEO) with accession number GSE144057. The mass spectrometry proteomics data have been deposited to the ProteomeXchange Consortium via the PRIDE partner repository with the dataset identifier PXD018000.

\section{Code availability}

Zentracker, the custom MATLAB software used to analyze locomotory responses is available at https:/github.com/wormtracker/zentracker. Neuron Analyzer, the customwritten MATLAB software used to analyze FRET levels (yellow cameleon), is available at https://github.com/neuronanalyser/neuronanalyser.

Received: 22 May 2019; Accepted: 26 March 2020;

Published online: 29 April 2020

\section{References}

1. Boulanger, L. M. Immune proteins in brain development and synaptic plasticity. Neuron 64, 93-109 (2009).

2. Deverman, B. E. \& Patterson, P. H. Cytokines and CNS development. Neuron 64, 61-78 (2009)

3. Stephan, A. H., Barres, B. A. \& Stevens, B. The complement system: an unexpected role in synaptic pruning during development and disease. Annu. Rev. Neurosci. 35, 369-389 (2012).

4. Goshen, I. et al. A dual role for interleukin-1 in hippocampal-dependent memory processes. Psychoneuroendocrinology 32, 1106-1115 (2007).

5. Stellwagen, D. \& Malenka, R. C. Synaptic scaling mediated by glial TNF- $\alpha$. Nature 440, 1054-1059 (2006).

6. Estes, M. L. \& McAllister, A. K. Immune mediators in the brain and peripheral tissues in autism spectrum disorder. Nat. Rev. Neurosci. 16, 469-486 (2015).

7. Iori, V., Frigerio, F. \& Vezzani, A. Modulation of neuronal excitability by immune mediators in epilepsy. Curr. Opin. Pharmacol. 26, 118-123 (2016)

8. Klein, R. S., Garber, C. \& Howard, N. Infectious immunity in the central nervous system and brain function. Nat. Immunol. 18, 132-141 (2017).

9. Miller, A. H. \& Raison, C. L. The role of inflammation in depression: from evolutionary imperative to modern treatment target. Nat. Rev. Immunol. 16, 22-34 (2016).

10. Pinho-Ribeiro, F. A., Verri, W. A. Jr \& Chiu, I. M. Nociceptor sensory neuron-immune interactions in pain and inflammation. Trends Immunol. 38, 5-19 (2017).

11. Ellwardt, E., Walsh, J. T., Kipnis, J. \& Zipp, F. Understanding the role of T cells in CNS homeostasis. Trends Immunol. 37, 154-165 (2016).

12. Schwartz, M. \& Deczkowska, A. Neurological disease as a failure of brain-immune crosstalk: the multiple faces of neuroinflammation. Trends Immunol. 37, 668-679 (2016).

13. Choi, G. B. et al. The maternal interleukin-17a pathway in mice promotes autism-like phenotypes in offspring. Science 351, 933-939 (2016).

14. Shin Yim, Y. et al. Reversing behavioural abnormalities in mice exposed to maternal inflammation. Nature 549, 482-487 (2017).

15. Segond von Banchet, G. et al. Neuronal IL-17 receptor upregulates TRPV4 but not TRPV1 receptors in DRG neurons and mediates mechanical but not thermal hyperalgesia. Mol. Cell. Neurosci. 52, 152-160 (2013).

16. Ebbinghaus, M. et al. Interleukin-17A is involved in mechanical hyperalgesia but not in the severity of murine antigen-induced arthritis. Sci. Rep. 7, 10334 (2017).

17. Chen, C. et al. IL-17 is a neuromodulator of Caenorhabditis elegans sensory responses. Nature 542, 43-48 (2017).

18. Staal, J. et al. Ancient origin of the CARD-Coiled coil/Bcl10/MALT1-like paracaspase signaling complex indicates unknown critical functions. Front. Immunol. 9, 1136 (2018).

19. Jaworski, M. \& Thome, M. The paracaspase MALT1: biological function and potential for therapeutic inhibition. Cell. Mol. Life Sci. 73, 459-473 (2016).

20. Ruefli-Brasse, A. A. Regulation of NF-B-dependent lymphocyte activation and development by paracaspase. Science 302, 1581-1584 (2003).
21. Ruland, J., Duncan, G. S., Wakeham, A. \& Mak, T. W. Differential requirement for Malt1 in $\mathrm{T}$ and $\mathrm{B}$ cell antigen receptor signaling. Immunity 19, 749-758 (2003)

22. Lundgren, D. H., Hwang, S.-I., Wu, L. \& Han, D. K. Role of spectral counting in quantitative proteomics. Expert Rev. Proteom. 7, 39-53 (2014).

23. Hachmann, J. et al. Mechanism and specificity of the human paracaspase MALT1. Biochem. J. 443, 287-295 (2012).

24. Uren, A. G. et al. Identification of paracaspases and metacaspases: two ancient families of caspase-like proteins, one of which plays a key role in MALT lymphoma. Mol. Cell 6, 961-967 (2000).

25. Hulpiau, P., Driege, Y., Staal, J. \& Beyaert, R. MALT1 is not alone after all: identification of novel paracaspases. Cell. Mol. Life Sci. 73, 1103-1116 (2016).

26. Couillault, C. et al. TLR-independent control of innate immunity in Caenorhabditis elegans by the TIR domain adaptor protein TIR-1, an ortholog of human SARM. Nat. Immunol. 5, 488-494 (2004).

27. Liberati, N. T. et al. Requirement for a conserved Toll/interleukin-1 resistance domain protein in the Caenorhabditis elegans immune response. Proc. Natl Acad. Sci. USA 101, 6593-6598 (2004).

28. Chuang, C. F. \& Bargmann, C. I. A Toll-interleukin 1 repeat protein at the synapse specifies asymmetric odorant receptor expression via ASK1 MAPKKK signaling. Genes Dev. 19, 270-281 (2005).

29. Inoue, A. et al. Forgetting in C. elegans is accelerated by neuronal communication via the TIR-1/JNK-1 pathway. Cell Rep. 3, 808-819 (2013).

30. Macosko, E. Z. et al. A hub-and-spoke circuit drives pheromone attraction and social behaviour in C. elegans. Nature 458, 1171-1175 (2009).

31. Laurent, P. et al. Decoding a neural circuit controlling global animal state in C. elegans. eLife 4, e04241 (2015).

32. Cabalzar, K. et al. Monoubiquitination and activity of the paracaspase MALT1 requires glutamate 549 in the dimerization interface. PLoS ONE 8, e72051-1 (2013).

33. Cheung, B. H. H., Arellano-Carbajal, F., Rybicki, I. \& de Bono, M. Soluble guanylate cyclases act in neurons exposed to the body fluid to promote C. elegans aggregation behavior. Curr. Biol. 14, 1105-1111 (2004).

34. Cheung, B. H. H., Cohen, M., Rogers, C., Albayram, O. \& de Bono, M. Experience-dependent modulation of $\mathrm{C}$. elegans behavior by ambient oxygen Curr. Biol. 15, 905-917 (2005).

35. Gray, J. M. et al. Oxygen sensation and social feeding mediated by a C. elegans guanylate cyclase homologue. Nature 430, 317-322 (2004).

36. Persson, A. et al. Natural variation in a neural globin tunes oxygen sensing in wild Caenorhabditis elegans. Nature 458, 1030-1033 (2009).

37. Rogers, C., Persson, A., Cheung, B. \& de Bono, M. Behavioral motifs and neural pathways coordinating $\mathrm{O} 2$ responses and aggregation in C. elegans. Curr. Biol. 16, 649-659 (2006).

38. Jaworski, M. et al. Malt1 protease inactivation efficiently dampens immune responses but causes spontaneous autoimmunity. EMBO J. 33, 2765-2781 (2014).

39. Gewies, A. et al. Uncoupling Malt1 threshold function from paracaspase activity results in destructive autoimmune Inflammation. Cell Rep. 9 1292-1305 (2014)

40. Bornancin, F. et al. Deficiency of MALT1 paracaspase activity results in unbalanced regulatory and effector $\mathrm{T}$ and $\mathrm{B}$ Cell responses leading to multiorgan inflammation. J. Immunol. 194, 3723-3734 (2015).

41. Lin, S.-C., Lo, Y.-C. \& Wu, H. Helical assembly in the MyD88-IRAK4-IRAK2 complex in TLR/IL-1R signalling. Nature 465, 885-890 (2010).

42. Qiao, Q. et al. Structural architecture of the CARMA1/Bcl10/ MALT1 signalosome: nucleation-induced filamentous assembly. Mol. Cell 51, 766-779 (2013)

43. Hayden, M. S. \& Ghosh, S. Shared principles in NF- $\kappa B$ signaling. Cell 132, 344-362 (2008)

44. Bannister, A. J. \& Kouzarides, T. The CBP co-activator is a histone acetyltransferase. Nature 384, 641-643 (1996).

45. Amatya, N., Garg, A. V. \& Gaffen, S. L. IL-17 signaling: The Yin and the Yang Trends Immunol. 38, 310-322 (2017)

46. Saeki, S., Yamamoto, M. \& Iino, Y. Plasticity of chemotaxis revealed by paired presentation of a chemoattractant and starvation in the nematode Caenorhabditis elegans. J. Exp. Biol. 204, 1757-1764 (2001).

47. Tan, M. W., Mahajan-Miklos, S. \& Ausubel, F. M. Killing of Caenorhabditis elegans by Pseudomonas aeruginosa used to model mammalian bacterial pathogenesis. Proc. Natl Acad. Sci. USA 96, 715-720 (1999).

48. de Bono, M. \& Bargmann, C. I. Natural variation in a neuropeptide Y receptor homolog modifies social behavior and food response in C. elegans. Cell 94, 679-689 (1998).

49. Reddy, K. C., Andersen, E. C., Kruglyak, L. \& Kim, D. H. A polymorphism in npr-1 is a behavioral determinant of pathogen susceptibility in C. elegans. Science 323, 382-384 (2009).

50. Van Gilst, M. R., Hadjivassiliou, H., Jolly, A. \& Yamamoto, K. R. Nuclear hormone receptor NHR-49 controls fat consumption and fatty acid composition in C. elegans. PLoS Biol. 3, e53-1 (2005). 
51. Kim, D. H. et al. A conserved p38 MAP kinase pathway in Caenorhabditis elegans innate immunity. Science 297, 623-626 (2002).

52. Troemel, E. R. et al. p38 MAPK regulates expression of immune response genes and contributes to longevity in C. elegans. PLoS Genet. 2, e183-1 (2006).

53. Shivers, R. P., Kooistra, T., Chu, S. W., Pagano, D. J. \& Kim, D. H. Tissuespecific activities of an immune signaling module regulate physiological responses to pathogenic and nutritional bacteria in C. elegans. Cell Host Microbe 6, 321-330 (2009).

54. $\mathrm{Li}, \mathrm{S}$. et al. A map of the interactome network of the metazoan C. elegans. Science 303, 540-543 (2004).

55. Borovikova, L. V. et al. Vagus nerve stimulation attenuates the systemic inflammatory response to endotoxin. Nature 405, 458-462 (2000).

56. Chiu, I. M. et al. Bacteria activate sensory neurons that modulate pain and inflammation. Nature 501, 52-57 (2013).

57. Pavlov, V. A. \& Tracey, K. J. Neural regulation of immunity: molecular mechanisms and clinical translation. Nat. Neurosci. 20, 156-166 (2017).

58. Hoffman, C. \& Aballay, A. Role of neurons in the control of immune defense. Curr. Opin. Immunol. 60, 30-36 (2019).

59. Kim, D. H. \& Ewbank, J. J. Signaling in the innate immune response. WormBook 1-35 https://doi.org/10.1895/wormbook.1.83.2 (2018).

60. Sun, J., Singh, V., Kajino-Sakamoto, R. \& Aballay, A. Neuronal GPCR controls innate immunity by regulating noncanonical unfolded protein response genes. Science 332, 729-732 (2011).

61. Styer, K. L. et al. Innate immunity in Caenorhabditis elegans is regulated by neurons expressing NPR-1/GPCR. Science 322, 460-464 (2008).

62. Anderson, A., Laurenson-Schafer, H., Partridge, F. A., Hodgkin, J. \& McMullan, R. Serotonergic chemosensory neurons modify the C. elegans immune response by regulating G-protein signaling in epithelial cells. PLoS Pathog. 9, e1003787-17 (2013).

63. Kawli, T. \& Tan, M.-W. Neuroendocrine signals modulate the innate immunity of Caenorhabditis elegans through insulin signaling. Nat. Immunol. 9, 1415-1424 (2008).

64. Zugasti, O. \& Ewbank, J. J. Neuroimmune regulation of antimicrobial peptide expression by a noncanonical TGF- $\beta$ signaling pathway in Caenorhabditis elegans epidermis. Nat. Immunol. 10, 249-256 (2009).

65. Zhang, Y., Lu, H. \& Bargmann, C. I. Pathogenic bacteria induce aversive olfactory learning in Caenorhabditis elegans. Nature 438, 179-184 (2005).

66. Ryzhakov, G., Blazek, K. \& Udalova, I. A. Evolution of vertebrate immunity: sequence and functional analysis of the SEFIR domain family member Act1. J. Mol. Evol. 72, 521-530 (2011)

67. Park, H. H. et al. The death domain superfamily in intracellular signaling of apoptosis and inflammation. Annu. Rev. Immunol. 25, 561-586 (2007).

68. Novatchkova, M., Leibbrandt, A., Werzowa, J., Neubüser, A. \& Eisenhaber, F. The STIR-domain superfamily in signal transduction, development and immunity. Trends Biochem. Sci. 28, 226-229 (2003).

69. Phelan, J. D. et al. A multiprotein supercomplex controlling oncogenic signalling in lymphoma. Nature 560, 387-391 (2018).

70. Israël, L. \& Bornancin, F. Ways and waves of MALT1 paracaspase activation. Cell. Mol. Immunol. 15, 8-11 (2017).

71. Israël, L. et al. A CARD10-dependent tonic signalosome activates MALT1 paracaspase and regulates IL-17/TNF- $\alpha$-driven keratinocyte inflammation. J. Invest. Dermatol. 138, 2075-2079 (2018).

72. Jeltsch, K. M. et al. Cleavage of roquin and regnase- 1 by the paracaspase MALT1 releases their cooperatively repressed targets to promote TH17 differentiation. Nat. Immunol. 15, 1079-1089 (2014).

73. Uehata, T. et al. Malt1-induced cleavage of regnase-1 in CD4+ Helper T cells regulates immune activation. Cell 153, 1036-1049 (2013).

74. Staal, J. et al. T-cell receptor-induced JNK activation requires proteolytic inactivation of CYLD by MALT1. EMBO J. 30, 1742-1752 (2011).

75. Finger, F. et al. Olfaction regulates organismal proteostasis and longevity via microRNA-dependent signaling. Nat. Metab. 1, 350-359 (2019).

76. Yamamoto, M. et al. Regulation of Toll/IL-1-receptor-mediated gene expression by the inducible nuclear protein IкBC. Nature 430, 218-222 (2004).

77. Motoyama, M., Yamazaki, S., Eto-Kimura, A., Takeshige, K. \& Muta, T. Positive and negative regulation of nuclear factor- $\mathrm{kB}$-mediated transcription

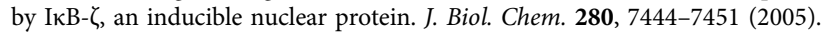

78. Reed, M. D. et al. IL-17a promotes sociability in mouse models of neurodevelopmental disorders. Nature 577, 249-253 (2020).

79. Minevich, G., Park, D. S., Blankenberg, D., Poole, R. J. \& Hobert, O. CloudMap: a cloud-based pipeline for analysis of mutant genome sequences. Genetics 192, 1249-1269 (2012).

80. Chen, C., Fenk, L. A. \& de Bono, M. Efficient genome editing in Caenorhabditis elegans by CRISPR-targeted homologous recombination. Nucleic Acids Res. 41, e193-e193 (2013).

81. Fenk, L. A. \& de Bono, M. Environmental CO2 inhibits Caenorhabditis elegans egg-laying by modulating olfactory neurons and evokes widespread changes in neural activity. Proc. Natl Acad. Sci. USA 112, E3525-E3534 (2015).

82. Zanin, E. et al. Affinity purifitcation of protein complexes in C. elegans. Methods Cell. Biol. 106, 289-322 (2011).

83. Cox, J. \& Mann, M. MaxQuant enables high peptide identification rates, individualized p.p.b.-range mass accuracies and proteome-wide protein quantification. Nat. Biotechnol. 26, 1367-1372 (2008).

84. Mata-Cabana, A., Sin, O., Seinstra, R. \& Nollen, E. Nuclear/cytoplasmic fractionation of proteins from Caenorhabditis elegans. Bio Protoc. 8, 1-15 (2018).

85. Hendriks, G.-J., Gaidatzis, D., Aeschimann, F. \& Großhans, H. Extensive oscillatory gene expression during C. elegans larval development. Mol. Cell 53, 380-392 (2014).

86. Reimand, J. et al. g:Profiler-a web server for functional interpretation of gene lists (2016 update). Nucleic Acids Res. 44, W83-W89 (2016).

87. Bargmann, C. I., Hartwieg, E. \& Horvitz, H. R. Odorant-selective genes and neurons mediate olfaction in C. elegans. Cell 74, 515-527 (1993).

88. Jeong, D.-E. et al. Mitochondrial chaperone HSP-60 regulates anti-bacterial immunity via p38 MAP kinase signaling. EMBO J. 36, 1046-1065 (2017).

89. Yang, J.-S. et al. OASIS: online application for the survival analysis of lifespan assays performed in aging research. PLoS ONE 6, e23525-1 (2011).

90. Artan, M. et al. Food-derived sensory cues modulate longevity via distinct neuroendocrine insulin-like peptides. Genes Dev. 30, 1047-1057 (2016).

\section{Acknowledgements}

We thank the Caenorhabditis Genetics Center (funded by National Institutes of Health Infrastructure Program P40 OD010440) and the Japanese knockout consortium for strains, the Cambridge Research Institute Genomics Core for whole genome sequencing, Adeline Colussi and Harvey McMahon for reagents, and de Bono lab members for comments on the manuscript. This work was supported by the Medical Research Council UK, the European Research Council (Advanced Grant 269058 to M.d.B), and Wellcome (209504/Z/17/Z Investigator Award to M.d.B.).

\section{Author contributions}

S.F., C.C., M.A., S.B., and M.d.B. conceived experiments; S.F., C.C., M.A., and S.B performed experiments; A.C. and G.N. performed sequence analysis; S.-Y.P.-C., F.B., and M.S. performed mass spectrometry analysis; S.F. and M.d.B. wrote the manuscript.

\section{Competing interests}

The authors declare no competing interests.

\section{Additional information}

Supplementary information is available for this paper at https://doi.org/10.1038/s41467020-15872-y.

Correspondence and requests for materials should be addressed to M.d.B.

Peer review information Nature Communications thanks Thorsten Hoppe and the other, anonymous, reviewer(s) for their contribution to the peer review of this work. Peer reviewer reports are available.

Reprints and permission information is available at http://www.nature.com/reprints

Publisher's note Springer Nature remains neutral with regard to jurisdictional claims in published maps and institutional affiliations.

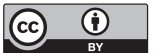

Open Access This article is licensed under a Creative Commons Attribution 4.0 International License, which permits use, sharing, adaptation, distribution and reproduction in any medium or format, as long as you give appropriate credit to the original author(s) and the source, provide a link to the Creative Commons license, and indicate if changes were made. The images or other third party material in this article are included in the article's Creative Commons license, unless indicated otherwise in a credit line to the material. If material is not included in the article's Creative Commons license and your intended use is not permitted by statutory regulation or exceeds the permitted use, you will need to obtain permission directly from the copyright holder. To view a copy of this license, visit http://creativecommons.org/ licenses/by/4.0/.

(c) The Author(s) 2020 